\title{
Fundamental Thermal Noise Limits for Optical Microcavities
}

\author{
Christopher Panuski, ${ }^{1, *}$ Dirk Englund, ${ }^{1}$ and Ryan Hamerly $\oplus^{1,2, \dagger}$ \\ ${ }^{1}$ Research Laboratory of Electronics, MIT, Cambridge, Massachusetts 02139, USA \\ ${ }^{2}$ NTT Research, Inc. Physics and Informatics Laboratories, \\ 940 Stewart Drive, Sunnyvale, California 94085, USA
}

(Received 19 May 2020; revised 15 September 2020; accepted 21 October 2020; published 7 December 2020)

\begin{abstract}
We present a joint theoretical and experimental analysis of thermorefractive noise in high-quality-factor $(Q)$, small-mode-volume $(V)$ optical microcavities. Analogous to well-studied stability limits imposed by Brownian motion in macroscopic Fabry-Perot resonators, we show that microcavity thermorefractive noise gives rise to a mode-volume-dependent maximum effective quality factor. State-of-the-art fabricated microcavities are found to be within one order of magnitude of this bound. By measuring the first thermodynamically limited frequency noise spectra of wavelength-scale high- $Q / V$ silicon photonic crystal cavities, we confirm the assumptions of our theory, demonstrate a broadband sub $-\mu \mathrm{K} / \sqrt{\mathrm{Hz}}$ temperature sensitivity, and unveil a new technique for discerning subwavelength changes in microcavity mode volumes. To illustrate the immediate implications of these results, we show that thermorefractive noise limits the optimal performance of recently proposed room-temperature, all-optical qubits using cavityenhanced bulk material nonlinearities. Looking forward, we propose and analyze coherent thermo-optic noise cancellation as one potential avenue toward violating these bounds, thereby enabling continued development in quantum optical measurement, precision sensing, and low-noise integrated photonics.
\end{abstract}

DOI: $10.1103 /$ PhysRevX.10.041046

\section{INTRODUCTION}

Room-temperature, high-quality-factor $(Q)$ optical cavities enable the investigation of new physical phenomena by enhancing light-matter interaction [1], shaping electromagnetic modes [2], and modifying the vacuum photon density of states [3]. However, these advantages come with an often forgotten cost-interaction with a thermally equilibrated confining medium inherently injects noise into the optical mode in accordance with the fluctuation-dissipation theorem (FDT) [4]. Macroscopic resonators [Fig. 1(a)], such as those implemented in gravitational wave interferometers, minimize this interaction by supporting a large mode volume $\tilde{V} \gg 1$ in vacuum, where $\tilde{V}=$ $V /(\lambda / n)^{3}$ for the volume $V$ of a $\lambda$-wavelength optical mode confined in a refractive index $n$. The surprising realization that the sensitivity of these kilometer-scale cavities can still be limited by Brownian motion in few-micron-thick mirror coatings $[5,6]$ has spurred interest in low-noise mirror coatings [7], grating-based mirrors [8,9], and the

\footnotetext{
cpanuski@mit.edu

rhamerly@mit.edu
}

Published by the American Physical Society under the terms of the Creative Commons Attribution 4.0 International license. Further distribution of this work must maintain attribution to the author(s) and the published article's title, journal citation, and DOI.
Subject Areas: Photonics, Statistical Physics

fundamental limits of macroscopic cavities in the presence of thermal fluctuations [10-12].

Here, we consider the opposite case: optical microcavities [Fig. 1(b)] [13], whose small mode volumes $(\tilde{V} \sim 1)$ facilitate single-molecule label-free sensing [14], highrepetition-rate frequency combs $[15,16]$ for frequency synthesis [17], spectroscopy [18], and astronomy [19], enhanced coupling for atom-photon interfaces [20-22], and low-energy (down to single-photon level) nonlinear interactions [23]. These microcavity-enhanced nonlinearities even reveal new directions in physical science [24]: experimental demonstrations of non-Hermitian phenomena [25], topological enhancement [26,27], synchronization [28], and chaotic dynamics [29] are just a few recent examples. However, shrinking mode confinement toward the near-diffraction-limited volumes offered by microcavities significantly amplifies fundamental temperature fluctuations $\left\langle\delta T^{2}\right\rangle \propto 1 / V$ [30]. These small-volume temperature fluctuations are a classic problem in statistical mechanics [31], and their exact nature was heavily debated [32-34] until initial measurements were reported in the 1990s [35]. Temperature noise has since been studied in diverse contexts including high-energy collisions [36], molecular dynamics [37], spin ensembles [38], and state-of-the-art electron- [39] or graphene-based nanocalorimeters [40]. In optics, the associated refractive index fluctuations, so-called "thermorefractive noise" (TRN), have been studied extensively in fiber-based lasers and interferometers [41-44]. In recent 
(a) Low- $Q / V$

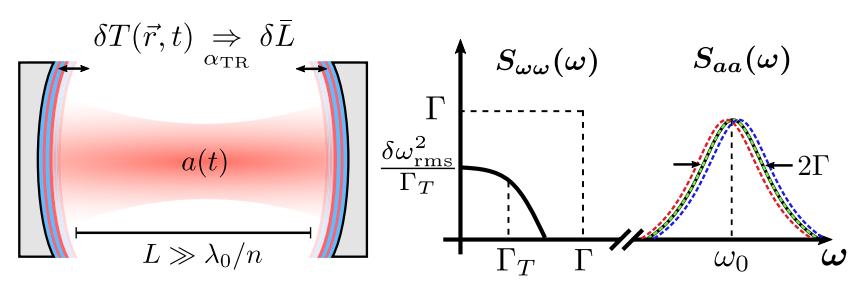

(b) High- $Q / V$

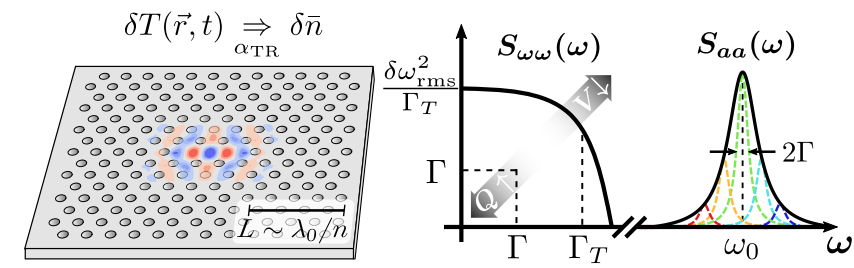

FIG. 1. Comparison of thermorefractive noise (TRN) in macroscopic resonators (a) and microcavities (b). Mode-averaged temperature fluctuations $\delta \bar{T}$ in large cavities induce refractive index noise $\delta \bar{n}$ (and, thus, path length changes $\delta \bar{L}$ ) due to the mirrors' nonzero thermo-optic coefficient $\alpha_{\mathrm{TR}}=d n / d T$. The large mode volume $V$ reduces $\delta \bar{T}$, yielding a narrow-band resonant frequency noise spectrum $S_{\omega \omega}(\omega)$ and an rms resonant frequency fluctuation $\delta \omega_{\text {rms }} \ll \Gamma$, the cavity half-linewidth. This nondominant thermal noise inhomogeneously broadens the intracavity field spectrum $S_{a a}(\omega)$. Decreasing $V$ increases both the magnitude $\delta \omega_{\text {rms }}$ and bandwidth $\Gamma_{T}$ of TRN, while increasing the resonator quality factor $Q=\omega_{0} / 2 \Gamma$ causes both quantities to exceed $\Gamma$. TRN, therefore, becomes a dominant source of homogeneous broadening in wavelength-scale high- $Q / V$ microcavities, leading to thermal dephasing and reduced resonant excitation efficiency of the cavity field $a(t)$.

years, TRN has emerged as a principal source of resonant frequency noise in various dielectric microcavity geometries including microspheres [45], whispering-gallery-mode resonators [46,47], ring resonators [48], and photonic crystal $(\mathrm{PhC})$ cavities [49]. TRN has also recently been shown to limit the stability of integrated lasers $[50,51]$ and microcavity frequency combs [52].

To date, microcavity TRN has been considered only in a perturbative regime, where the resulting $\mathrm{rms}$ resonant frequency fluctuation $\delta \omega_{\text {rms }} \underset{\sim}{\propto} 1 / \sqrt{V}$ is much less than the loaded cavity linewidth $\widetilde{2} \Gamma=\omega_{0} / Q$. For sufficiently high $Q$ and small $V$, this assumption becomes invalid. Continued improvements in microcavity performanceyielding $Q>10^{7}, \tilde{V} \sim 1$ through fabrication advances [53] and $Q \sim 10^{5}, \tilde{V} \sim 10^{-3}$ using novel subwavelength dielectric features [54] — thus raises a simple question: when will fundamental thermal noise limit the performance of high- $Q / V$ microcavities?

Here, we answer this open question by deriving general bounds for optical microcavity performance in the presence of TRN and find that current photonic crystal and whispering-gallery-mode (WGM) devices are within one order of magnitude of this bound. We verify our theory by measuring TRN as the dominant noise source in high- $Q / V \mathrm{PhC}$ cavities and demonstrate the ability to distinguish between subwavelength mode volumes $(\tilde{V}<1)$ using fundamental noise spectra. To our knowledge, these are the first spectrally resolved measurements of a near-diffractionlimited optical mode operating at the thermal noise limit. We believe that our devices' unique combination of micron-scale spatial localization with a broadband temperature sensitivity comparable to state-of-the-art room-temperature optical thermometers will enable new directions in thermal physics and nonequilibrium thermodynamics $[55,56]$. As an example of the immediate impact of our formalism, we analyze the implications for an outstanding goal in quantum photonics: all-optical qubits using cavityenhanced bulk material nonlinearities [57]. Since thermal noise is found to limit the qubit coherence, we propose and analyze coherent thermo-optic noise cancellation as one potential avenue toward continued developments in lownoise, high- $Q / V$ microcavities. Together, these results reveal the importance of thermal noise in state-of-the-art optical resonators, inform design choices to minimize its impact on device performance, and motivate new research directions to violate the proposed bounds.

\section{FORMALISM}

As schematically illustrated in Fig. 1, fundamental stochastic temperature fluctuations $\delta T(\vec{r}, t)$ within a cavity confining medium of refractive index $n$ and thermo-optic coefficient $\alpha_{\mathrm{TR}}=d n / d T$ drive a mode-averaged refractive index change $\delta \bar{n}(t)=\alpha_{\mathrm{TR}} \delta \bar{T}(t)$. For an optical mode completely confined in dielectric, the resulting resonance shift $\delta \omega(t)=-\omega_{0} \alpha_{\mathrm{TR}} \delta \bar{T}(t) / n$ follows from first-order perturbation theory [58]. For now, we neglect temperature-induced deformations of the cavity, as the thermoelastic coefficient of common dielectrics is typically 2 orders of magnitude smaller than $\alpha_{\mathrm{TR}}$ [46]. Resonant enhancement of these mechanical effects over a narrow bandwidth is possible; however, we are primarily interested in the broadband noise performance.

In the presence of TRN, the steady-state rotating-frame intracavity field amplitude is

$$
\tilde{a}(t)=\sqrt{\Gamma} \tilde{s}_{\text {in }} \int_{-\infty}^{t} d t^{\prime} e^{-(i \Delta+\Gamma)\left(t-t^{\prime}\right)+i \int_{t^{\prime}}^{t} d t^{\prime \prime} \delta \omega\left(t^{\prime \prime}\right)}
$$

for a loaded amplitude decay rate $\Gamma$ and critically coupled static drive $\tilde{s}_{\text {in }}$ detuned by $\Delta$ from the cavity resonance. The associated statistical moments can be computed using the moment-generating properties of the characteristic functional $\left\langle e^{i \int_{t^{\prime}}^{t} \delta \omega\left(t^{\prime \prime}\right) d t^{\prime \prime}}\right\rangle$, which in the case of zero-mean Gaussian noise requires only the autocorrelation $\langle\delta \omega(t) \delta \omega(t+\tau)\rangle=\left(\omega_{0} \alpha_{\mathrm{TR}} / n\right)^{2}\langle\delta \bar{T}(t) \delta \bar{T}(t+\tau)\rangle[59,60]$. The latter autocorrelation of temperature fluctuations can be computed from the heat equation 


$$
\frac{\partial}{\partial t} \delta T(\vec{r}, t)+D_{T} \nabla^{2} \delta T(\vec{r}, t)=F_{T}(\vec{r}, t)
$$

in a medium of thermal diffusivity $D_{T}$ driven by a Langevin forcing term $F_{T}(\vec{r}, t)$ which satisfies the FDT. As we illustrate for slab PhC cavities, Eq. (2) can be solved analytically for specific geometries; however, for generality, we follow the approach of Ref. [61] and enforce a single-mode decay approximation by introducing a phenomenological thermal decay rate

$$
\Gamma_{T}=D_{T} \frac{\int\left\{\nabla\left[\epsilon(\vec{r})|\vec{E}(\vec{r})|^{2}\right]\right\}^{2} d^{3} \vec{r}}{\int \epsilon(\vec{r})^{2}|\vec{E}(\vec{r})|^{4} d^{3} \vec{r}}
$$

evaluated for the envelope of intracavity energy density. This form of $\Gamma_{T}$ is chosen for consistency with $\left\langle\delta \bar{T}^{2}\right\rangle=k_{B} T_{0}^{2} / c_{V} V_{T}$, the well-known statistical mechanics result for temperature fluctuations in a volume $V_{T}$ of specific heat capacity $c_{V}$ at thermal equilibrium with a bath temperature $T_{0}$ [31]. Averaging Eq. (2) over the optical mode profile, we then find

$$
\frac{d}{d t} \delta \bar{T}(t)+\Gamma_{T} \delta \bar{T}(t)=\bar{F}_{T}(t)
$$

leading to the solution

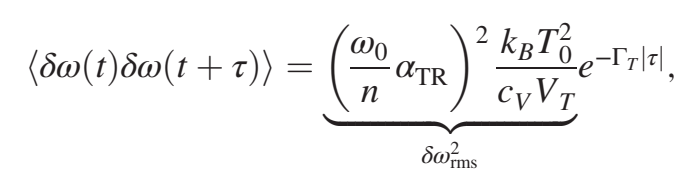

where the thermal mode volume

$$
V_{T}=\frac{\left[\int \epsilon(\vec{r})|\vec{E}(\vec{r})|^{2} d^{3} \vec{r}\right]^{2}}{\int \epsilon(\vec{r})^{2}|\vec{E}(\vec{r})|^{4} d^{3} \vec{r}}
$$

is the common Kerr nonlinear mode volume found by solving Eq. (2) in a homogeneous medium [45,62]. For a three-dimensional mode with a Gaussian-shaped energy density distribution, $V_{T}$ is larger than the standard Purcell mode volume $V=\int \epsilon|\vec{E}|^{2} d^{3} \vec{r} / \max \left\{\epsilon|\vec{E}|^{2}\right\}$ by a factor of $2 \sqrt{2}$.

Combining Eqs. (1) and (5), we can solve for the statistical moments of the driven cavity amplitude $\tilde{a}(t)$ as a function of the primary parameters $V_{T}$ and $\Gamma_{T}$. The results are discussed in Secs. IV and V based on the complete derivations in Appendix A. Here, however, we consider a simplified example of free cavity evolution, which demonstrates the basic techniques and the cavity behavior in two limiting regimes. In this case, the amplitude autocorrelation $\left\langle\tilde{a}(t) \tilde{a}^{*}(t+\tau)\right\rangle \sim\left\langle e^{-i \int_{t}^{t+\tau} \delta \omega\left(t^{\prime}\right) d t^{\prime}}\right\rangle e^{-\Gamma \tau}=$ $F(\tau) e^{-\Gamma \tau}$ can be simplified by evaluating the dephasing function

$$
\begin{aligned}
F(\tau) & =\exp \left[-\int_{0}^{\tau} d \tau^{\prime}\left(\tau-\tau^{\prime}\right)\langle\delta \omega(t) \delta \omega(t+\tau)\rangle\right] \\
& =\exp \left[\left(\frac{\delta \omega_{\mathrm{rms}}}{\Gamma_{T}}\right)^{2}\left(1-\Gamma_{T} \tau-e^{-\Gamma_{T} \tau}\right)\right]
\end{aligned}
$$

where we implement the previously described characteristic functional properties with the frequency noise statistics of Eq. (5). For sufficiently large $V_{T}$ (corresponding to a slow $\Gamma_{T}$ ) and small $Q$ as assumed in previous analyses, the cavity resonance is quasistatic over the photon decay period and shifts by much less than a cavity linewidth over time. In this perturbative limit [Fig. 1(a)], $\Gamma_{T} \tau \ll 1$ over the cavity ringdown time. We can therefore expand $e^{-\Gamma_{T} \tau}$ in Eq. (7) to second order, yielding $\left\langle\tilde{a}(t) \tilde{a}^{*}(t+\tau)\right\rangle \sim e^{-\Gamma \tau} e^{-\delta \omega_{\mathrm{m} s}^{2} \tau^{2} / 2}$. The associated line shape, given by the Fourier transform of $\left\langle\tilde{a}(t) \tilde{a}^{*}(t+\tau)\right\rangle$ via the Wiener-Khinchin theorem, is then the original Lorentzian with a small inhomogeneous Gaussian broadening due to TRN. Simply put, TRN limits the cavity stability without substantially altering the intracavity dynamics.

However, as the thermal mode volume $V_{T}$ shrinks, $\delta \omega_{\text {rms }}$ and $\Gamma_{T}$ increase until they eventually exceed $\Gamma$ [Fig. 1(b)]. In this high- $Q / V$ limit, the resonant frequency $\omega_{0}(t)$ directly tracks the temperature noise over the relevant timescales [i.e., the frequency noise in Eq. (5) is effectively $\delta$ correlated], leading to a homogeneous broadening of the resonance that dephases the intracavity field. Inserting the associated limit $\Gamma_{T} \tau \gg 1$ into Eq. (7), we find $\left\langle\tilde{a}(t) \tilde{a}^{*}(t+\tau)\right\rangle \sim e^{-\left(\Gamma+\delta \omega_{\mathrm{ms}}^{2} / \Gamma_{T}\right) \tau}$, corresponding to the broadened linewidth $2 \Gamma+2 \delta \omega_{\mathrm{rms}}^{2} / \Gamma_{T} \approx 2 \delta \omega_{\mathrm{rms}}^{2} / \Gamma_{T}$.

Our analysis focuses on the transition to this high- $Q / V$ limit. Specifically, we derive general solutions to Eq. (1) for arbitrary TRN powers and bandwidths to calculate mode-volume-dependent maximum "effective" cavity quality factors $Q_{\text {eff }}$ that describe the fundamental limits of microcavity stability and coherence. Whereas the simple free evolution model presented in this section reveals the essential physics of the extreme low- and high- $Q / V$ cases, our latter generalized solutions are broadly applicable to any highly confined dielectric microcavity, including microspheres [45], micropillars [63], ring resonators [48], microtoroids [64], PhC cavities [49], microdisks [65], vertical Fabry-Perot cavities [66], and more.

\section{TRN MEASUREMENT}

Before pursuing these goals, we first experimentally verify the fundamental assumptions of our TRN model by measuring the noise spectrum of high- $Q / V$ PhC cavities. As shown in Fig. 2, our setup uses a Mach-Zehnder interferometer to measure the phase of a cavity reflection signal via balanced homodyne detection. A variable beam splitter separates the emission from an amplified tunable infrared laser into local oscillator (LO) and cavity input paths, which are passively balanced to minimize laser 


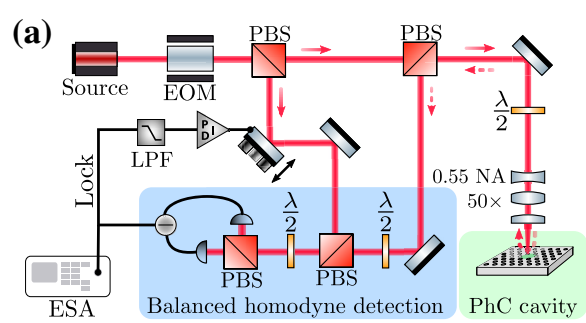

(b)

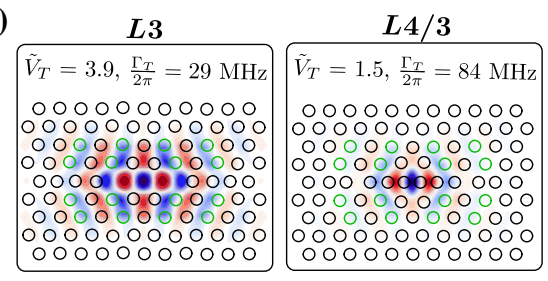

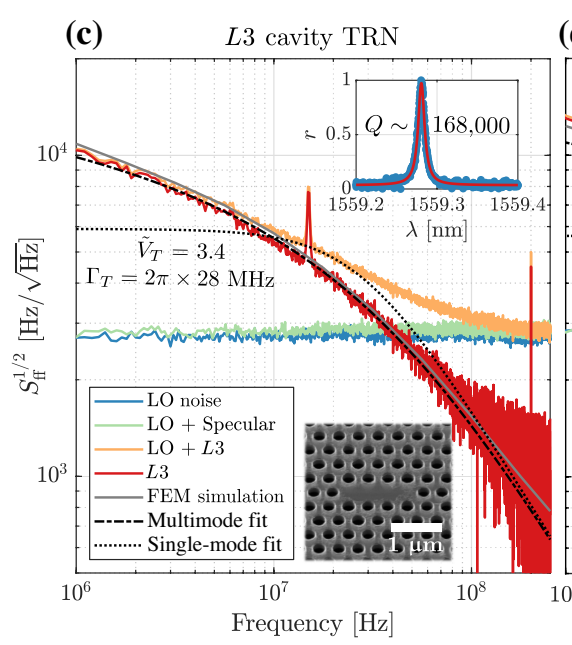

(d)

$L 4 / 3$ cavity TRN

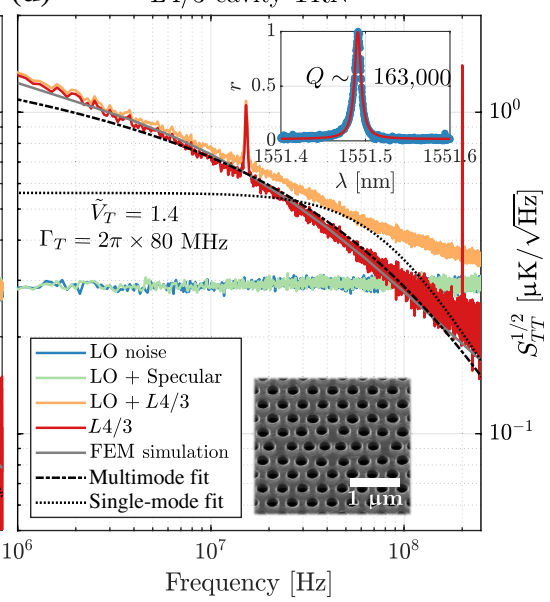

FIG. 2. Calibrated measurement of TRN in high- $Q / V$ silicon PhC cavities. A shot-noise-limited, balanced homodyne detector (a) is locked to the phase quadrature of the cavity reflection signal and records the spectrum of resonant frequency fluctuations. The finite difference time domain (FDTD) simulated mode profiles, thermal mode volume $\tilde{V}_{T}$ [Eq. (6)], and thermal decay rate $\Gamma_{T}$ [Eq. (3)] of the $L 3$ and $L 4 / 3$ devices tested are shown in (b). The radii of the green holes are increased by up to $5 \%$ to form superimposed gratings which improve vertical coupling efficiency. The measured spectral density of cavity resonant frequency and temperature noise- $S_{\mathrm{ff}}(f)$ and $S_{\mathrm{TT}}(f)$, respectively - (red) for $L 3$ (c) and $L 4 / 3$ (d) cavities are compared to noise from a specular reflection off the sample surface, finite-element method (FEM) simulations of cavity TRN, as well as single- and multimode fits. The listed multimode fit parameters agree with the predicted values in (b). Inset reflection spectra of each device reveal quality factors on the order of $10^{5}$, which compare favorably with the FDTD computed values $\left(1.7 \times 10^{5}\right.$ and $1.6 \times 10^{5}$ for the $L 4 / 3$ and $L 3$, respectively). Micrographs of the fabricated designs with enlarged holes relative to the optimal designs in (b) are also inset.

frequency noise coupling. A $\lambda / 2$ plate rotates the input signal polarization by $45^{\circ}$ relative to the dominant cavity polarization axis such that the cavity reflection can be isolated from any specular reflection from the sample using a polarizing beam splitter (PBS) [67]. The sample stage is temperature controlled to better than $10 \mathrm{mK}$ using a Peltier plate and feedback temperature controller. A balanced, shot-noise-limited photodetector measures the homodyne signal from the recombined cavity reflection and LO, and the result is recorded on an electronic spectrum analyzer (ESA). By actively locking to the phase quadrature of the homodyne signal with a piezocontrolled mirror, TRNinduced cavity frequency noise is detected as frequencyresolved voltage noise. To calibrate the spectrum, we inject a known phase noise with an electro-optic modulator $(\mathrm{EOM})$ whose modulation efficiency is measured by sideband fitting [68].

Figure 2 shows the resulting measurements for two released (air-clad) silicon $\mathrm{PhC}$ cavities: the common $L 3$ cavity [69] and the recently proposed " $L 4 / 3$ " cavity [70]. Fabricated cavities yield high quality factors (up to $Q \approx$ 400000 at $\lambda_{0} \approx 1550 \mathrm{~nm}$ ) with efficient vertical coupling [71]. The variation of Purcell mode volume- $-\tilde{V}=$ $(0.95,0.32)$ for simulated $L 3$ and $L 4 / 3$ cavities, respectively-also allows us to confirm the expected volume dependence of TRN. Whereas a direct reflection from the sample surface (green trace) adds little additional noise to the LO background (blue), we observe broadband noise from either cavity's reflection (orange). The calibration tone is visible at $200 \mathrm{MHz}$, and we attribute the resonance at approximately $15 \mathrm{MHz}$ to optomechanical coupling from the fundamental flexural mode of the suspended membrane [72]. In the corrected cavity noise curve (red), we subtract the LO shot noise and account for attenuation due to the finite cavity linewidth. As expected, the wavelength-scale mode volumes yield a spectral density of resonant frequency fluctuations $S_{\mathrm{ff}}(f)$ with nearly 2 orders of magnitude larger amplitude and bandwidth compared to previous results in microspheres [45] and ring resonators [48]. The corrected $L 4 / 3$ noise spectra in Fig. 3 also confirm that the measurement is invariant across the range of acceptable input powers, which is limited below by the homodyne locking stability and above by the onset of nonlinear effects leading to excess noise. Calibration data, an extended description of the setup, and an analysis of other possible noise sources are provided in Appendix B.

The measured noise spectra show excellent agreement with numerical simulations based on a modified version of the fluctuation-dissipation theorem for thermorefractive noise [73], which is further described in Sec. VI. We also develop an analytic noise model based on a multimode solution to Eq. (2) in a thin slab, which is similarly well fitted to the data and yields the fit parameters $\left\{\tilde{V}_{T}^{L 3}, \Gamma_{T}^{L 3} / 2 \pi, \tilde{V}_{T}^{L 4 / 3}, \Gamma_{T}^{L 4 / 3} / 2 \pi\right\}=\{3.4 \pm 0.3,28 \pm 1 \mathrm{MHz}$, $1.4 \pm 0.1,80 \pm 3 \mathrm{MHz}\}$ that compare favorably with the expected values $(\{3.9,29 \mathrm{MHz}, 1.5,84 \mathrm{MHz}\})$ from 


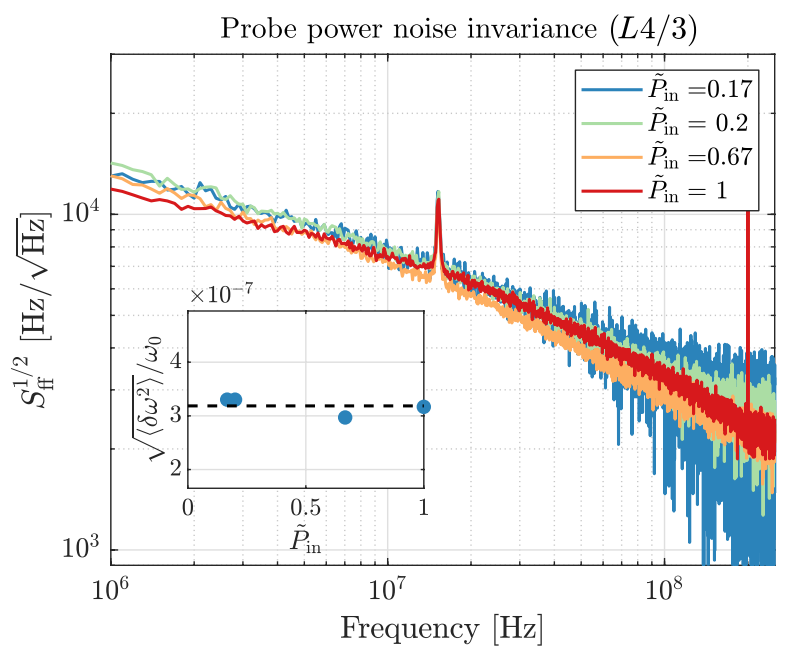

FIG. 3. Comparison of corrected $L 4 / 3$ cavity frequency noise spectra at various input powers $\tilde{P}_{\text {in }}$ normalized to the maximum value for the dataset. The rms fractional frequency fluctuation $\sqrt{\left\langle\delta \omega^{2}\right\rangle} / \omega_{0}$ is computed from the integrated noise over the plotted measurement bandwidth and plotted in the inset as a function of $\tilde{P}_{\text {in }}$. No significant power scaling or deviation from the mean value (black dashed line) is observed, indicating that noise contributions from nonlinear effects can be neglected.

Eqs. (6) (evaluated numerically from the finite element method-simulated mode profiles) and (3). In Eq. (3), we assume a two-dimensional Gaussian mode and reduced thermal diffusivity $D_{T}=D(1-\phi) /(1+\phi)$ for the patterned slab with porosity $\phi$ compared to the unpatterned thin film diffusivity $D[74,75]$. As predicted, the reduced mode volume of the $L 4 / 3$ cavity increases the bandwidth and spectral density of thermal fluctuations. These observations thereby illustrate a new technique for evaluating the mode volume of fabricated optical resonators using fundamental quantities as opposed to complex invasive techniques, such as near-field scanning optical microscopy [54].

The noise spectra of the proposed single-mode approximation [Eq. (5)] underestimate the measured noise of both devices at low frequencies $\omega \ll \Gamma_{T}$ but accurately approximate $S_{\mathrm{ff}}$ in the range of frequencies of interest (near and above the cutoff frequency $\Gamma_{T}$ ) and conserve the integrated frequency noise $\left\langle\delta \omega_{\text {rms }}^{2}\right\rangle$. Our results indicate that TRN is the dominant noise source in high- $Q / V$ resonators and validate the suitability of a single-mode approximation to describe the spectrum of frequency fluctuations in general microcavity geometries. To our knowledge, these measurements are the first demonstration of broadband, wavelength-scale cavity readout at the thermodynamic limit. The corresponding temperature sensitivity, $S_{T T}^{1 / 2} \sim$ $300 \mathrm{nK} / \sqrt{\mathrm{Hz}}$ as shown in Fig. 2, is within one order of magnitude of room-temperature records set by multimode WGM thermometers $[76,77]$. Compared to those state-ofthe-art sensors, our $\mathrm{PhC}$ devices occupy 6 orders of magnitude less area and offer 3 orders of magnitude larger bandwidth. We expect this unique combination of micronscale spatial resolution and broadband, thermodynamically limited readout to enable new directions in thermal physics.

\section{MICROCAVITY $Q / V$ LIMITS}

Having experimentally verified the TRN model, we can now use it to estimate the fundamental performance limits of room-temperature microcavities. As described in Sec. II, TRN broadens the cavity linewidth to $2 \Gamma_{\text {eff }}$ according to the cavity mode volume $V_{T}$ and thermal decay rate $\Gamma_{T}$. The associated quality factor $Q_{\text {eff }}=\omega_{0} / 2 \Gamma_{\text {eff }}$ is therefore bound to a maximum value indicative of the resonance stability, which, in turn, determines the fidelity of integrated optical frequency references or synthesizers [17], the minimum resolvable resonance shift in microcavity sensors [78], and the spectral purity of microcavity lasers [79]. We also specifically consider the quality factor to mode volume ratio $Q_{\text {eff }} / V$, which is proportional to the peak intracavity intensity and, therefore, of particular significance for cavity nonlinear optics [80] and enhanced sensitivity to pointlike defects [81].

The effective quality factor $Q_{\text {eff }}=\omega_{0}\left\langle|\tilde{a}(t)|^{2}\right\rangle / 2\left|\tilde{s}_{\text {in }}\right|^{2}$ of interest in this case can alternatively be viewed as the ratio of intracavity energy $\left\langle|\tilde{a}(t)|^{2}\right\rangle$ to energy input per cycle $2\left|\tilde{s}_{\text {in }}\right|^{2} / \omega_{0}$ in a resonantly excited, critically coupled cavity. Under the same conditions, solving Eq. (1) for $\left\langle|\tilde{a}(t)|^{2}\right\rangle$ subject to the noise autocorrelation of Eq. (5) yields

$$
\frac{Q_{\mathrm{eff}}}{\tilde{V}}=\frac{\omega_{0}}{2 \Gamma_{T} \tilde{V}} e^{x} x^{-s} \gamma_{l}(s, x),
$$

where $\gamma_{l}$ is the lower incomplete Gamma function, $x=\left(\delta \omega_{\mathrm{rms}} / \Gamma_{T}\right)^{2}$, and $s=\Gamma / \Gamma_{T}+x$. Intuitively, decreasing the cavity linewidth $2 \Gamma$ well below the broadened linewidth has little impact: the prolonged energy storage offsets the reduced excitation rate of the rapidly shifting resonance, leaving the intracavity energy unaltered. $Q_{\text {eff }}$ is maximized in this limiting case. The corresponding upper bound of Eq. (8) at $T=300 \mathrm{~K}$ is plotted for various material systems in Fig. 4 as a function of $\tilde{V}$ assuming a three-dimensional Gaussian-shaped mode in a homogeneous three- or two-dimensional confining medium. In the latter case, the decay rate $\Gamma_{T}=3 \pi D_{T} / V^{2 / 3}$ decreases by a factor of $3 \sqrt{2} V^{1 / 3}$ to account for the restricted dimensionality of thermal diffusion.

In the joint limit $\left(\delta \omega_{\mathrm{RMS}}, \Gamma\right) \ll \Gamma_{T}$, valid for sufficiently high- $Q$, low- $V$ cavities, Eq. (8) simplifies to $Q_{\text {eff }}^{\max }=$ $\omega_{0} \Gamma_{T} / 2 \delta \omega_{\text {rms }}^{2}$, thereby recovering the broadened linewidth $2 \delta \omega_{\mathrm{rms}}^{2} / \Gamma_{T}$ derived in Sec. II. $Q_{\mathrm{eff}}^{\max }$ then scales as $V^{1 / 3}$ in a homogeneous medium, indicating that larger mode volumes reduce the integrated thermo-optic noise, as expected. For this reason, recent ultrahigh- $Q\left(Q>10^{8}\right)$ integrated resonators are specifically designed with $\tilde{V} \gg 1$ to limit 


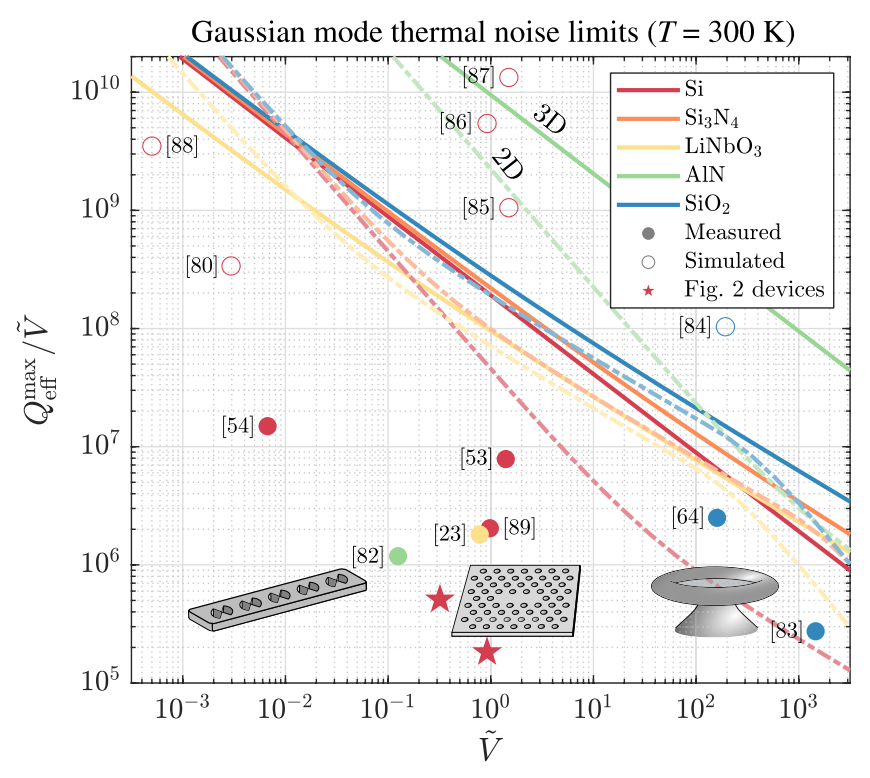

FIG. 4. Thermal-noise-limited room-temperature quality factor to mode volume ratios $\left(Q_{\mathrm{eff}}^{\max } / \tilde{V}\right)$ for the materials considered in Appendix $C$ assuming a Gaussian-shaped mode at $\lambda_{0}=1550 \mathrm{~nm}$ admitting thermal diffusion in two or three dimensions (dashdotted and solid lines, respectively). These limits are compared our devices as well as other fabricated and proposed microcavities. Insets illustrate typical confinement geometries for the range of $\tilde{V}$ listed (see Refs. [23,53,54,64,80,82-89]).

TRN [65,90]. Alternatively, Fig. 4 illustrates the advantage of reducing $V$ to maximize $Q_{\text {eff }}^{\max } / V$. Further optimization of subwavelength cavities $[54,91]$ could therefore improve the intensity enhancement achievable in room-temperature devices toward the open goal of microcavity-based quantum nonlinear optics.

Surprisingly, the $Q_{\mathrm{eff}}^{\max } / \tilde{V}$ limits of several common materials lie within an order of magnitude. Yet this effect is not fundamental: aluminum nitride, for example, is shown to outperform all other plotted materials by over an order of magnitude due to its simultaneously large thermal conductivity and small thermo-optic coefficient. While our review of high- $Q / V$ cavities (Fig. 4) in various materials shows that all fabricated cavities obey the projected bounds, silicon $\mathrm{PhC}$ slab cavities [53] and silica microtoroids [64] lie within an order of magnitude of the thermal noise limit. Furthermore, various simulated devices [84-87] exceed the limit; their practical realization thereby requires low-temperature operation-where TRN and the thermo-optic coefficient are both suppressed [92]—or novel noise-suppression techniques as discussed in Sec. VI.

\section{IMPLICATIONS FOR ALL-OPTICAL QUBITS}

These proposed thermal noise limits have practical impact for future devices. Chief among the applications driving the pursuit for high- $Q / V$ cavities is quantum information. Within the past year alone, numerous proposals [93-97] have explored the feasibility of photonic microcavity-based quantum gates using strong photon-photon interactions mediated by bulk material nonlinearities. Driven by recent developments in high- $Q / V$ microcavities $[54,98]$ and thin film nonlinear optical materials, current experiments are approaching 1\% [99] of this so-called "qubit limit of cavity nonlinear optics" [57] where single-photon nonlinearities outpace cavity losses. Strong emitter-based single-photon nonlinearities in high- $Q / V$ cavities are also a promising route toward optically addressable qubits but rely on precise coupling to single atoms [100], ions [22], quantum dots [101], or defect centers [102]. We therefore focus our analysis on emitter-free, all-optical qubits using bulk nonlinearities. These techniques promise room-temperature operation-the requisite hallmark for connecting distant nodes in future quantum networks [103] — by leveraging the relative immunity of optical photons to thermal noise. While this insensitivity is granted by Planck's law, we show here that, through the thermorefractive effect, temperature fluctuations can significantly impact light in a high- $Q / V$ resonator. For coherent processes, TRN-induced dephasing of the field amplitude $\tilde{a}(t)$ must be considered in addition to the previously discussed stability and intracavity energy limitations in Sec. IV. We therefore solve for $\langle\tilde{a}(t)\rangle$ in the presence of TRN. This result corresponds to an effective quality factor $Q_{\text {eff }}=\omega_{0}|\langle\tilde{a}(t)\rangle|^{2} / 2\left|\tilde{s}_{\text {in }}\right|^{2}$ in a resonant, critically coupled cavity, yielding

$$
Q_{\mathrm{eff}}=\frac{\omega_{0}}{2 \Gamma_{\mathrm{eff}}}=Q\left(\frac{\Gamma}{\Gamma_{T}}\right)^{2} e^{2 x} x^{-2 s} \gamma_{l}^{2}(s, x)
$$

for the previously defined $x$ and $s$. Equation (9) describes the coherence of the intracavity field and is synonymous with the dephasing time $T_{2}^{*}=1 / \Gamma_{\text {eff }}=2 Q_{\text {eff }} / \omega_{0}$ commonly considered for quantum emitters [104].

We can compare $\Gamma_{\text {eff }}$ to a nonlinear coupling rate $g$ between qubit basis states with the simple figure of merit $\mathrm{FOM}=g / 2 \Gamma_{\text {eff }}$, which intuitively corresponds to the number of qubit operations that can be completed prior to decay or dephasing. For bulk $\chi^{(3)}$ and $\chi^{(2)}$ nonlinearities, the coupling rate $g$ is a function of material parameters and mode volumes [105]. In the case of $\chi^{(3)}$ interactions, $g=$ $\omega_{0} K_{\chi} / \tilde{V}_{\text {Kerr }}$ for the Kerr interaction mode volume $\tilde{V}_{\text {Kerr }}=$ $\tilde{V}_{T}$ (as discussed in Sec. II) and coupling constant

$$
K_{\chi}=\frac{3 \pi \hbar c}{2 n \epsilon_{0}} \frac{\chi^{(3)}}{\lambda_{0}^{4}}
$$

at wavelength $\lambda_{0}$, where $\hbar, c$, and $\epsilon_{0}$ are Planck's constant, the speed of light, and the vacuum permittivity, respectively. In the strong coupling regime $g / 2 \Gamma \gg 1$, the intensity-dependent refractive index leads to an anharmonicity of Fock state energies that decouples the qubit 

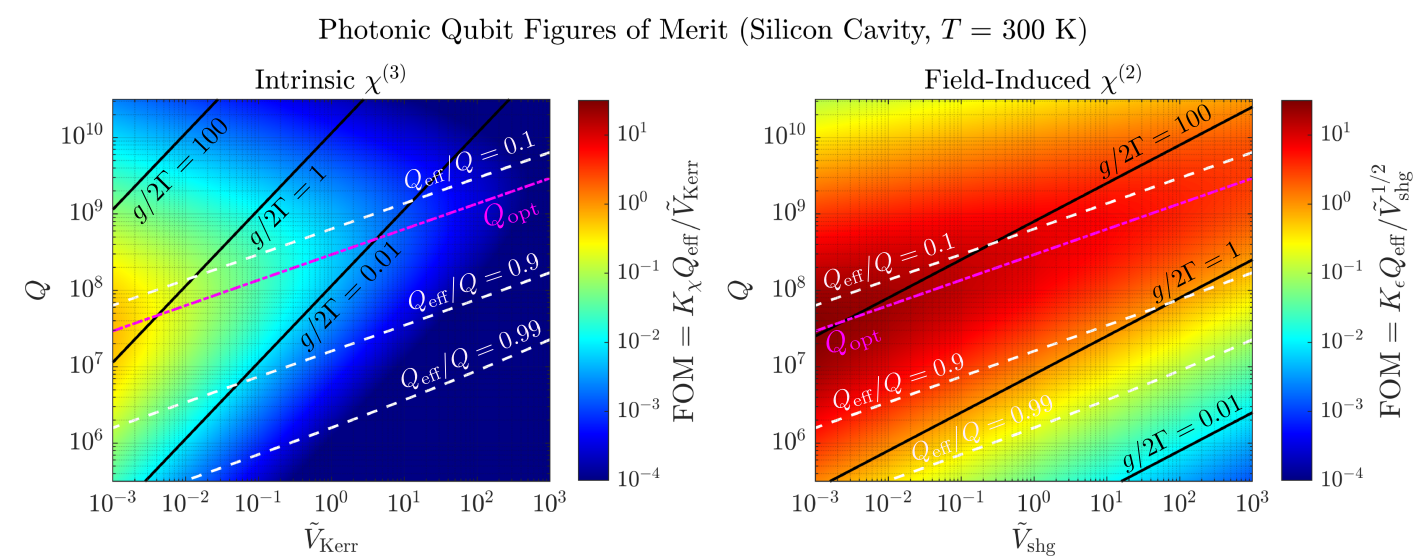

FIG. 5. Performance of room-temperature all-optical qubits using bulk $\chi^{(3)}$ (left) or electric-field-induced $\chi^{(2)}$ (right) nonlinearities in silicon microcavities as a function of loaded cavity quality factor $Q$ at $\lambda_{0}=2.3 \mu \mathrm{m}$ and the relevant normalized nonlinear mode volume $\tilde{V}\left(\tilde{V}_{\text {Kerr }}=\tilde{V}_{T}\right.$ and $\tilde{V}_{\text {shg }}$, assumed to be equal to $\tilde{V}_{T}$, for $\chi^{(3)}$ and $\chi^{(2)}$, respectively). The figure of merit (FOM)-the ratio of qubit coupling rate $g$ to the composite decay and thermal dephasing rate $2 \Gamma_{\text {eff }}=\omega_{0} / Q_{\text {eff }}$ is largest for strong coupling $(g / 2 \Gamma \gg 1)$ and weak dephasing $\left(Q_{\text {eff }} \approx Q\right)$. These competing characteristics yield an optimum quality factor $Q=Q_{\text {opt }}$ for any $\tilde{V}$. Three-dimensional thermal diffusion in a homogeneous medium is assumed.

basis (zero- and one-photon states) from higher-energy states [57].

Alternatively, bulk $\chi^{(2)}$ nonlinearities can mediate coupling between doubly resonant first- and second-harmonic qubit basis states. The coupling rate $g=\omega_{0} K_{\epsilon} / \tilde{V}_{\text {shg }}^{1 / 2}$ then describes the frequency of Rabi oscillations between a single photon in the second-harmonic mode and two photons at the fundamental frequency [93,106,107]. Assuming an equal amplitude decay rate $\Gamma$ for both modes, strong coupling again requires $g / 2 \Gamma \gg 1$. Here, the applicable coupling constant and second-harmonic mode volume are defined as

$$
\begin{gathered}
K_{\epsilon}=\sqrt{\frac{\pi \hbar c}{n^{3} \epsilon_{0}}} \frac{\chi^{(2)}}{\lambda^{2}}, \\
V_{\text {shg }}=\frac{\left(\int n^{2}\left|\vec{E}_{2 \omega}\right|^{2} d^{3} \vec{r}\right)\left(\int n^{2}\left|\vec{E}_{\omega}\right|^{2} d^{3} \vec{r}\right)^{2}}{\left|\int_{*} n^{3} E_{2 \omega}^{*(i)} E_{\omega}^{(j)} E_{\omega}^{(k)} d^{3} \vec{r}\right|^{2}}
\end{gathered}
$$

for the first- and second-harmonic modes $\vec{E}_{\omega}$ and $\vec{E}_{2 \omega}$, respectively, and the indices $i, j$, and $k$ corresponding to field components associated with the dominant $\chi^{(2)}$ tensor element. The integral $\int(\ldots) d^{3} \vec{r}$ is taken over all space, while $\int_{*}(\ldots) d^{3} \vec{r}$ is restricted to the nonlinear material. Extended derivations of $K_{\chi}, K_{\varepsilon}, V_{\text {shg }}$, and $V_{\text {Kerr }}$ are included in Appendix D.

The resulting figures of merit

$$
\mathrm{FOM}_{\chi^{(3)}}=K_{\chi} \frac{Q_{\text {eff }}}{\tilde{V}_{\text {Kerr }}}, \quad \mathrm{FOM}_{\chi^{(2)}}=K_{\epsilon} \frac{Q_{\text {eff }}}{\tilde{V}_{\text {shg }}^{1 / 2}}
$$

are plotted in Fig. 5 for Gaussian-shaped modes in silicon. Similar figures of merit are applicable to high-fidelity single-photon sources [105,107]. While silicon's centrosymmetric structure precludes an intrinsic $\chi^{(2)}$ nonlinearity, we assume the intrinsic $\chi^{(3)}$ nonlinearity can create an electric-field-induced $\chi^{(2)}=3 \chi^{(3)} E_{\mathrm{dc}}$ near the breakdown dc electric field $E_{\mathrm{dc}}$ [108].

An ideal qubit operates well within the strong coupling regime with minimal dephasing. In the presence of TRN, increasing $Q / V$ improves the former at the cost of the latter, leading to the observed mode-volume-dependent optimum loaded quality factor $Q_{\mathrm{opt}} \approx \omega_{0} \Gamma_{T} / 2 \delta \omega_{\text {rms. }}^{2}$. Figure 5 also illustrates a relative performance advantage for $\chi^{(2)}$ devices in silicon, as strong coupling can be achieved at lower quality factors. For example, the peak $\mathrm{FOM}_{\chi^{(2)}} \sim 10$ is 3 orders of magnitude greater than $\mathrm{FOM}_{\chi^{(3)}}$ assuming $Q=$ $Q_{\text {opt }}$ and $\tilde{V}_{\text {Kerr }}=\tilde{V}_{\text {shg }}=1$. Although small $\tilde{V}_{\text {shg }}$-which as illustrated in Eq. (12) involves maximizing the nonlinear overlap function between two colocalized cavity modesis generally more difficult to achieve than small $\tilde{V}_{\text {Kerr }}$ [109], $\mathrm{FOM}_{\chi^{(2)}} \propto \tilde{V}_{\text {shg }}^{-1 / 2}$ also demonstrates more favorable scaling at larger mode volumes.

\section{BEYOND THE THERMAL NOISE LIMIT}

This brief example manifests the practical limitations imposed by TRN, which yield wavelength-scale ultrahigh$Q$ resonators [65], narrow-linewidth lasers [110], and highperformance optical qubits thermodynamically forbidden. Breaking this fundamental "noise-volume trade-off" in optical microcavities would lift our proposed $Q / V$ limits and could enable quantum-noise-limited readout of diffraction-limited optical modes, thereby unveiling new possibilities in physics. Active cavity stabilization is one candidate solution; however, the limited speed of control 
loops and tuning mechanisms has restricted the noise rejection bandwidth of previous demonstrations to a few hundred hertz [76]. Passive feedback noise rejection via photothermal backaction [61]—resonance-detuningdependent laser heating that counteracts temperature fluctuations - has also been experimentally demonstrated [52], but the noise reduction scales with $\Gamma_{T}^{-1 / 2}$ and therefore becomes ineffective for small cavities. Furthermore, neither feedback-based technique can suppress fluctuations in the high- $Q / V$ regime, where the rate of resonant frequency fluctuations exceeds that of cavity leakage.

To overcome these limitations, we propose and analyze coherent thermo-optic (TO) noise cancellation as an avenue toward the open goal of broadband thermal noise suppression. This technique stems from an analogous proposal for thermal noise reduction in mirror coatings [7,30,111-113]: when the uniform temperature of a thickness $t$ coating is raised by $\delta T$, the increase in phase accumulation $\delta \phi=2 \pi t \delta n / \lambda=2 \pi t \alpha_{\mathrm{TR}} \delta T / \lambda$ from an increase in refractive index $\delta n$ can be offset by the coating expansion $\delta t=t \alpha_{\mathrm{TE}}^{L} \delta T$ toward the incident beam provided the linear coefficient of thermal expansion $\alpha_{\mathrm{TE}}^{L}$ and $\alpha_{\mathrm{TR}}$ have the same sign. In a microcavity, this relationship is reversed. Intuitively, we expect thermooptic noise to be minimized when the normalized thermoelastic (TE) frequency shift $\delta \tilde{\omega}_{\mathrm{TR}}=\delta \omega_{\mathrm{TE}} / \omega=$ $-\alpha_{\mathrm{TE}}^{L} \delta T$ of a freely expanding cavity equals that of the thermorefractive (TR) effect, $\delta \tilde{\omega}_{\mathrm{TR}}=-\alpha_{\mathrm{TR}} \delta T / n$, leading to the athermal condition

$$
\frac{\alpha_{\mathrm{TE}}^{L}}{\alpha_{\mathrm{TR}}}=-\frac{\gamma}{n}
$$

for the energy confinement fraction $\gamma \in(0,1]$ of the optical mode in the dielectric. Equation (14) can be satisfied by tuning $\gamma$ provided $\alpha_{\mathrm{TE}}^{L}$ and $\alpha_{\mathrm{TR}}$ are of comparable magnitude with opposite sign. Whereas we previously assumed $\left|\alpha_{\mathrm{TE}}^{L} / \alpha_{\mathrm{TR}}\right| \ll 1$ for common dielectrics, these two requirements are remarkably well satisfied by a range of polymers, where the Clausius-Mossotti relation dictates $\alpha_{\mathrm{TE}}^{L} / \alpha_{\mathrm{TR}} \approx$ $-\left(n^{2}+2\right)\left(n^{2}-1\right) \quad$ [114]. In polymethyl-methacrylate (PMMA, $n=1.48$ ), for example, $\gamma=0.83$ enables steady-state athermal operation. Our previously demonstrated high- $Q / V$ polymer "ladder" cavity designs [115] schematically illustrated in Fig. 6(a) are therefore well suited for TO noise cancellation.

But does steady-state athermal behavior imply complete broadband TO noise cancellation? Unfortunately, the answer is no, which we illustrate with the computational form of the FDT used to simulate TRN in Sec. III [5,73]. In this formalism, the total TO resonance shift

$$
\delta \tilde{\omega}_{\mathrm{TO}}=\int \underbrace{\left[g_{\mathrm{TR}}(\vec{r})+g_{\mathrm{TE}}(\vec{r})\right]}_{g_{\mathrm{TO}}(\vec{r})} \delta T(\vec{r}) d^{3} \vec{r},
$$

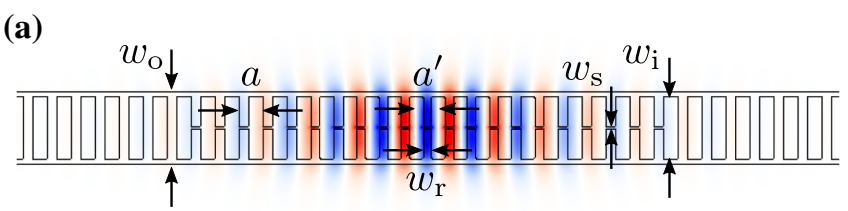

(b)

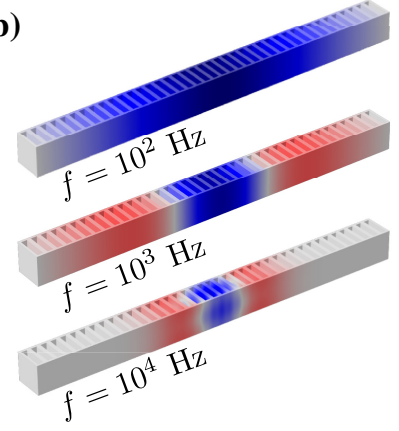

(c) (d)

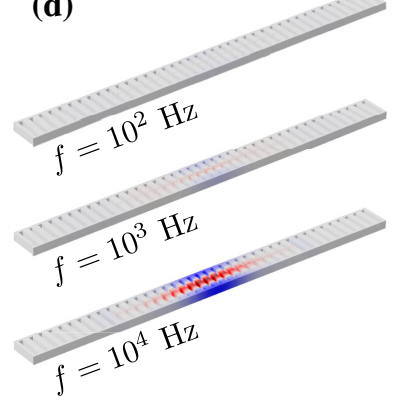

(e) Optimized

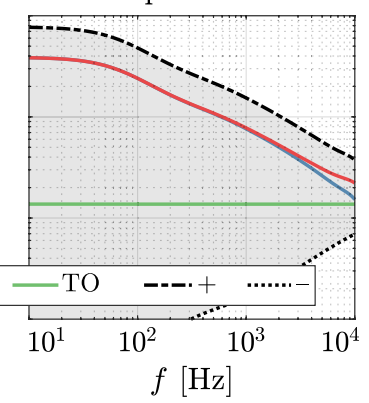

FIG. 6. Athermal polymer (PMMA) microcavity design by coherent thermo-optic noise cancellation. The $Q$-optimized baseline ladder-type nanobeam cavity (a) with $\left\{a, a^{\prime}, w_{o}, w_{i}, w_{s}\right\}=$ $\left\{647 \mathrm{~nm}, 0.914 a, 3 a, 0.85 w_{o}, 0\right\}$ and thickness $t=3 a$ is formed by quadratically tapering the lattice constant $a$ to $a^{\prime}$ over the inner 12 periods with a constant "rung" duty cycle $\eta_{r}=w_{r} / a=0.4$. This cavity is driven by anticorrelated volumetric and boundary heat sources corresponding to TR and TE noise, respectively, in FEM-based fluctuation-dissipation simulations. The composite TO frequency noise spectrum $S_{\mathrm{ff}} \propto[\nabla \delta T(\vec{r}, f)]^{2}$ is computed from the resulting harmonic temperature profiles $\delta T(\vec{r}, f)$ (b). The TO noise level is bounded by the coherent (-) and incoherent $(+)$ sum of TR and TE contributions (shaded region). The optimized parameters $\left\{a, a^{\prime}, w_{o}, w_{i}, w_{s}, t, \eta_{r}\right\}=\left\{697 \mathrm{~nm}, 0.874 a, 3 a, 0.85 w_{o}\right.$, $10 \mathrm{~nm}, 0.8 a, 0.6\}$ provide broadband TON cancellation (e) as visually depicted by reduced $\delta T(\vec{r}, f)$ at low frequencies (d). The color scale in (d) is equal to (b) to facilitate comparison.

i.e., the spatial average of temperature fluctuations $\delta T(\vec{r})$ weighted by $g_{\mathrm{TO}}(\vec{r})$, is calculated at frequency $\omega_{i}$ by driving the entropy $S$ (conjugate to $\delta T$ ) with a harmonic, perturbative heat source $\delta Q_{\mathrm{TO}}(\vec{r}, t)=T \delta S(\vec{r}, t)=$ $T Q_{0} g_{\mathrm{TO}}(\vec{r}) \cos \left(\omega_{i} t\right)$, where $Q_{0}$ is a constant of proportionality. The resulting spectrum of fluctuations,

$$
S_{\omega \omega}\left(\omega_{i}\right)=\frac{2 k_{B} T}{\pi \omega_{i}^{2} Q_{0}^{2}} W_{\mathrm{diss}},
$$

at the equilibrium temperature $T$ is then computed from the time-averaged $(\langle\cdot\rangle)$ power dissipation [73] 


$$
W_{\mathrm{diss}}=\int \frac{\kappa}{T}\left\langle[\nabla \delta T(\vec{r}, t)]^{2}\right\rangle d^{3} \vec{r}
$$

due to irreversible heat flow from the resulting harmonic temperature profile $\delta T(\vec{r}, t)$ in a material with thermal conductivity $\kappa$. We obtain the functional form of the TR and TE contributions to the composite TO weight function $g_{\mathrm{TO}}(\vec{r})=g_{\mathrm{TR}}(\vec{r})+g_{\mathrm{TE}}(\vec{r})$ by placing the associated firstorder perturbation theory results $[58,116]$ into the form of Eq. (15):

$$
\begin{aligned}
& \delta \tilde{\omega}_{\mathrm{TR}} \approx \int_{\Omega} \underbrace{-\alpha_{\mathrm{TR}}(\vec{r}) n^{2}|\vec{E}(\vec{r})|^{2}}_{g_{\mathrm{TR}}(\vec{r})} \delta T(\vec{r}) d^{3} \vec{r}, \\
& \delta \tilde{\omega}_{\mathrm{TE}} \approx \int_{\partial \Omega}-\underbrace{-\frac{\alpha_{\mathrm{TE}}^{L} h(\vec{r})}{4\left(n^{2}-1\right)^{-1}}\left[\left|\vec{E}_{\|}(\vec{r})\right|^{2}+\frac{\left|\vec{D}_{\perp}(\vec{r})\right|^{2}}{n^{2}}\right]}_{g_{\mathrm{TE}}(\vec{r})} \delta T(\vec{r}) d^{2} \vec{r} .
\end{aligned}
$$

Here, $\vec{E}_{\|}\left(\vec{D}_{\perp}\right)$ is the electric (electric displacement) field parallel (perpendicular) to the cavity boundary $\partial \Omega, h(\vec{r})$ is the dielectric thickness between neighboring boundaries, and we assume a normalized mode $\int n^{2}|\vec{E}|^{2} d^{3} \vec{r}=1$. We also note that Eq. (19) is valid only for sufficiently low frequencies $\omega \ll D_{T} / \max \{h(\vec{r})\}^{2}$ such that $\delta T(\vec{r})$ is approximately uniform throughout the dielectric between neighboring boundaries. We therefore see that TRN is associated with an energy-density-dependent volumetric heat source $\delta Q_{\mathrm{TR}}(\vec{r})=T Q_{0} g_{\mathrm{TR}}(\vec{r})$, whereas TE noise is associated with a surface heat source $\delta Q_{\mathrm{TE}}(\vec{r})=$ $T Q_{0} g_{\mathrm{TE}}(\vec{r})$ that depends on the field intensity and mechanical displacement amplitude of the dielectric boundary. Given this formulation, we immediately see that the TO noise cannot be completely canceled at all frequencies due to the spatial mismatch between the heat sources, which invariably leads to irreversible heat flow and the associated noise. However, our analysis reveals two intuitive design principles to maximize the coherence between anticorrelated TR and TE sources, thereby minimizing the total TO noise: (i) The cavity boundaries should be placed near highintensity regions of the cavity mode to maximize the spatial overlap between TR and TE sources; (ii) we intuitively expect that the amplitude of the harmonic temperature profile, and thereby the TO noise, should be minimized if the total added heat $Q_{\mathrm{TO}}=\int_{\Omega} \delta Q_{\mathrm{TR}}(\vec{r}) d^{3} \vec{r}+\int_{\partial \Omega} \delta Q_{\mathrm{TE}}(\vec{r}) d^{2} \vec{r}$ is zero. This expectation is true away from the sources at sufficiently low frequencies, where thermal diffusion effectively "masks" the exact source locations, and is actually equivalent to the athermal condition of Eq. (14).

Guided by these two simple principles, we optimized the coherent TO noise cancellation of the PMMA ladder cavity in Fig. 6. Our baseline design yields $Q=3 \times 10^{5}, \tilde{V}=2.6$, and $\gamma=0.75$-approximately $10 \%$ away from the athermal confinement condition. However, the beam ends are assumed to be fixed for support, which restricts lateral thermal expansion and leads to a dominant TR effect as shown by the individual TR and TE noise spectra in Fig. 6(c). If both heat sources are simultaneously simulated, the resulting TO noise resembles the coherent combination of the individual contributions; i.e., the noise amplitudes subtract as desired. However, as depicted by the associated harmonic temperature profiles in Fig. 6(b), the achievable noise reduction is modest owing to the dominant amplitude of the TR source near the center, high-intensity region of the cavity mode.

According to criterion (ii), it is evident that the TE source must be amplified to improve the noise cancellation. Freeing the cavity ends achieves this goal by enabling isotropic expansion but violates criterion (i): expansion along the beam length amplifies boundary displacements and the associated TE heat source away from the TR source maximum at the cavity center, thereby minimizing the spatial overlap between the two heat sources. As a result, TO noise suppression is again limited despite the nearly equal TR and TE noise contributions.

To simultaneously colocate the heat sources and magnify the TE effect, we instead propose placing a thin air slot through the ladder rungs at the center of the cavity as shown in Fig. 6(a). In the original design, expansion along the ladder rung length displaces the side rail boundary, where the cavity field is weak. The modified design's narrow slot moves this displacement boundary to the center of the beam (the "half-rungs" expand to fill the gap), where the cavity field and TR heat source are maximized. We then thin the beam to satisfy criterion (ii) within $1 \%$ and redesign the cavity defect region to maximize $Q$. The final optimized design supports a high- $Q / V$ resonance $\left(Q=6 \times 10^{4}\right.$, $\tilde{V}=0.75$ ) that suppresses fundamental thermal noise by over an order of magnitude at low frequencies. The temperature profiles in Fig. 6(e) graphically evidence this cancellation and suggest again that the achievable suppression is limited by the imperfect coherence of the two spatially resolved heat sources.

This intuition-driven optimization is a first proof-ofconcept demonstration of broadband, coherent cancellation of fundamental thermodynamic fluctuations in a microcavity. While the achievable noise reduction in our trial structure is relatively limited, the design is based on simple principles that can be readily extended to other designs, materials, and optimization techniques. For example, the interdisciplinary thermo-optical design of high- $Q / V$, lownoise resonators requires structural modifications that are well suited to recent developments in topological optimization of subwavelength integrated photonics $[91,117,118]$. The coherent thermo-optic cancellation scheme can also be applied other materials beyond polymers, such as CMOS-compatible titanium dioxide $\left(\mathrm{TiO}_{2}\right)$ [119] or perovskites [120] with negative thermo-optic coefficients. Our 
preliminary results thus motivate an exciting new research avenue into novel noise abatement schemes in pursuit of ultrahigh- $Q / V$ cavities beyond the thermal noise limit.

\section{SUMMARY AND OUTLOOK}

Ultimately, understanding the fundamental stability and coherence limits of optical microcavities relies on the proper characterization of thermo-optic noise. Toward this end, we have presented a general theory for thermorefractive noise in optical microcavities, discussed the resulting practical limitations on future integrated photonic components, and highlighted design choices that optimize device performance in its presence. We experimentally verified our model by measuring the dominant effect of temperature fluctuations in high- $Q / V$ silicon $\mathrm{PhC}$ cavities, which demonstrated the viability of optical microcavities as high-spatial-resolution temperature probes operating at the fundamental thermal noise limit. Our results show that nonperturbative TRN ultimately limits the achievable quality factor in small-mode-volume cavities and that experimental devices have neared this fundamental bound. Violating the observed trade-off between mode volume and thermo-optic noise stands as an exciting avenue for future investigation. We hope that our proposal and analysis of one possible solution-coherent thermo-optic noise cancellation-toward low-noise, high- $Q / V$ integrated optical devices further motivates this field of research. Ultimately, these advances will be necessary to achieve the performance required for future developments in optical quantum information processing, cavity optomechanics, precision optical sensing, and beyond.

\section{ACKNOWLEDGMENTS}

The authors thank M. Dykman, G. Huang, and T. Kippenberg for useful discussions. C. P. was supported by the Hertz Foundation Elizabeth and Stephen Fantone Family Fellowship and the MIT Jacobs Presidential Fellowship. R. H. was supported by an IC postdoctoral fellowship at MIT, administered by Oak Ridge Institute for Science and Education through U.S. DoE/ODNI. Experiments were supported in part by AFOSR Grant No. FA9550-16-1-0391, supervised by G. Pomrenke.

\section{APPENDIX A: TRN THEORY}

While the development of ultrahigh-performance optical resonators has only recently warranted its study within optical systems, stochastic temperature fluctuations are a fundamental concept in thermodynamics [31]. Assuming Boltzmann statistics within a finite volume $V$ with specific heat capacity $c_{V}$ at temperature $T$, we find

$$
\left\langle\delta T^{2}\right\rangle=\frac{k_{B} T^{2}}{c_{V} V} .
$$

In optical microcavities, $V$ approaches diffraction-limited volumes leading to temperature fluctuations that significantly impact the resonance stability in materials with a temperature-dependent refractive index.

Here, we derive the associated TRN spectrum in an optical microcavity under the single-mode approximation described in the main text. Using this approximation, the intracavity field statistics are derived. In the typical perturbative limit where the rms frequency fluctuation is much smaller than the loaded cavity linewidth $\left(\delta \omega_{\text {rms }} \ll 2 \Gamma_{l}\right)$, we use perturbation theory to solve for the evolution of the cavity field $a(t)$ and the associated noise spectrum $S_{a a}(t)$. We also provide general solutions for the first and second statistical moments of $a(t)$, which are used in the main text to derive "effective" quality factors in the presence of thermal noise. The solution for $S_{a a}(t)$ in the limiting case of high- $Q$ cavities-where the thermal decay rate $\Gamma_{T} \gg \Gamma_{l}$ and the frequency noise can be assumed to be white-is also provided. Finally, we compare the single-mode noise spectrum to that derived from a formal solution to heat diffusion in an infinite two-dimensional slab, which we find to most accurately model the specific geometry of the photonic crystal microcavities in our experiments.

A few notes on convention: (i) We derive two-sided angular frequency noise spectra $S_{\omega \omega}(\omega)$ but plot one-sided frequency spectra $S_{\mathrm{ff}}(f)=2 S_{\omega \omega}(2 \pi f) / 2 \pi=S_{\omega \omega}(2 \pi f) / \pi$ for experimental measurements to conform with the common conventions of the gravitational wave community; (ii) temporal coupled mode theory decay rates $\Gamma_{i}$ are amplitude decay rates; the associated quality factors are therefore defined as $Q_{i}=\omega_{0} / 2 \Gamma_{i}$.

\section{Statistics of microcavity TRN}

To first order, the change in resonant frequency under a permittivity perturbation $\delta \epsilon(\vec{r}, t)$ can be expressed as

$$
\begin{aligned}
\frac{\delta \omega(t)}{\omega_{0}} & =-\frac{1}{2} \frac{\int_{\delta \epsilon} \delta \epsilon(\vec{r}, t)|\vec{E}(\vec{r})|^{2} d^{3} \vec{r}}{\int \epsilon|\vec{E}(\vec{r})|^{2} d^{3} \vec{r}} \\
& \approx-\frac{1}{V_{\text {eff }}} \frac{\int_{\delta n} n \delta n(\vec{r}, t)|\vec{E}(\vec{r})|^{2} d^{3} \vec{r}}{\max \left\{\epsilon|\vec{E}(\vec{r})|^{2}\right\}},
\end{aligned}
$$

where we make the approximation $\delta \epsilon \approx 2 n \delta n$ and introduce the standard mode volume

$$
V_{\mathrm{eff}}=\frac{\int \epsilon(\vec{r})|\vec{E}(\vec{r})|^{2} d^{3} \vec{r}}{\max \left\{\epsilon(\vec{r})|\vec{E}(\vec{r})|^{2}\right\}}
$$

The change in refractive index $\delta n(\vec{r}, t)$ is directly proportional to temperature change $\delta T(\vec{r}, t)$, with the thermo-optic coefficient $\alpha_{\mathrm{TR}}=d n / d T$ serving as the constant of proportionality. We therefore find 


$$
\frac{\delta \omega(t)}{\omega_{0}}=-\frac{1}{V_{\mathrm{eff}}} \frac{\int_{\delta n} \alpha_{\mathrm{TR}} \delta T(\vec{r}, t) n|\vec{E}(\vec{r})|^{2} d^{3} \vec{r}}{\max \left\{\epsilon|\vec{E}(\vec{r})|^{2}\right\}}
$$

Alternatively, Eq. (A2) can be evaluated for a uniform, mode-averaged temperature change $\delta \bar{T}(t)$ assuming complete confinement of the mode within a homogeneous medium. This approach yields

$$
\frac{\delta \omega(t)}{\omega_{0}}=-\frac{1}{n} \alpha_{\mathrm{TR}} \delta \bar{T}(t) .
$$

Comparing Eqs. (A4) and (A5), we find

$$
\delta \bar{T}(t)=\frac{1}{V_{\mathrm{eff}}} \frac{\int \delta T(\vec{r}, t) \epsilon(\vec{r})|\vec{E}(\vec{r})|^{2} d^{3} \vec{r}}{\max \left\{\epsilon|\vec{E}(\vec{r})|^{2}\right\}} .
$$

Equation (A5) can now be solved using the mode-averaged temperature change, whose evolution is derived from the heat equation (given a thermal diffusivity $D_{T}$ )

$$
\frac{\partial \delta T(\vec{r}, t)}{\partial t}-D_{T} \nabla^{2} \delta T=F_{T}(\vec{r}, t)
$$

driven by a thermal Langevin source $F_{T}(\vec{r}, t)$ with the statistics [121]

$$
\begin{aligned}
& \left\langle F_{T}\left(\vec{r}_{1}, t_{1}\right) F_{T}^{*}\left(\vec{r}_{2}, t_{2}\right)\right\rangle \\
& \quad=\frac{2 D_{T} k_{B} T_{0}^{2}}{c_{V}} \delta\left(t_{1}-t_{2}\right) \vec{\nabla}_{\vec{r}_{1}} \cdot \vec{\nabla}_{\vec{r}_{2}}\left[\delta\left(\vec{r}_{1}-\vec{r}_{2}\right)\right]
\end{aligned}
$$

that satisfy the fluctuation-dissipation theorem. Averaging over the mode, we find the approximation

$$
\frac{d[\delta \bar{T}(t)]}{d t}+\Gamma_{T} \delta \bar{T}(t)=\bar{F}_{T}(t)
$$

where $\Gamma_{T}$ is introduced as a phenomenological thermal decay rate whose form is chosen later to ensure the form of $\left\langle\delta \bar{T}^{2}\right\rangle$ matches the canonical result from statistical mechanics [Eq. (A1)]. In analog with $\delta \bar{T}(t)$, the mode-averaged thermal force $\bar{F}_{T}(t)$ is

$$
\bar{F}_{T}(t)=\frac{1}{V_{\text {eff }}} \frac{\int F_{T}(\vec{r}, t) \epsilon(\vec{r})|\vec{E}(\vec{r})|^{2} d^{3} \vec{r}}{\max \left\{\epsilon|\vec{E}(\vec{r})|^{2}\right\}} .
$$

The steady-state solution of Eq. (A9),

$$
\delta \bar{T}(t)=\int_{-\infty}^{t} \bar{F}\left(t^{\prime}\right) e^{-\Gamma_{T}\left(t-t^{\prime}\right)} d t^{\prime}
$$

can then be used to find the corresponding statistics of the temperature fluctuation at equilibrium (i.e., long $t$ ):

$$
\begin{aligned}
\langle\delta \bar{T}(t) \delta \bar{T}(t+\tau)\rangle= & \int_{-\infty}^{t} d t^{\prime} \int_{-\infty}^{t+\tau} d t^{\prime \prime}\left\langle\bar{F}_{T}\left(t^{\prime}\right) \bar{F}_{T}\left(t^{\prime \prime}\right)\right\rangle \\
& \times e^{-\Gamma_{T}\left(t-t^{\prime}\right)} e^{-\Gamma_{T}\left(t+\tau-t^{\prime \prime}\right)}
\end{aligned}
$$

The result requires the autocorrelation of $\bar{F}_{T}(t)$, which is readily evaluated using the mode-averaged form of Eq. (A10):

$$
\begin{aligned}
\left\langle\bar{F}_{T}(t) \bar{F}_{T}^{*}(t+\tau)\right\rangle & =\underbrace{\frac{2 D_{T} k_{B} T_{0}^{2}}{c_{V} V_{\text {eff }}^{2}} \frac{\int d^{3} \vec{r}\left\{\vec{\nabla}\left[\epsilon(\vec{r})|\vec{E}(\vec{r})|^{2}\right]\right\}^{2}}{\left[\max \left\{\epsilon|\vec{E}(\vec{r})|^{2}\right\}\right]^{2}} \delta(\tau)}_{\mathcal{R}_{\bar{F}_{T} \bar{F}_{T}}(0)} \\
& =\mathcal{R}_{\bar{F}_{T} \bar{F}_{T}}(0) \delta(\tau) .
\end{aligned}
$$

Inserting this result into Eq. (A12) along with the change of variables $t^{\prime \prime} \rightarrow t^{\prime}+\tau^{\prime}$ yields

$$
\langle\delta \bar{T}(t) \delta \bar{T}(t+\tau)\rangle \approx \frac{\mathcal{R}_{\bar{F}_{T} \bar{F}_{T}}(0)}{2 \Gamma_{T}} e^{-\Gamma_{T}|\tau|} .
$$

Correspondence with Eq. (A1) therefore requires

$$
\frac{\mathcal{R}_{\bar{F}_{T} \bar{F}_{T}}(0)}{2 \Gamma_{T}}=\frac{k_{B} T_{0}^{2}}{c_{V} V} \Rightarrow \mathcal{R}_{\bar{F}_{T} \bar{F}_{T}}(0)=\frac{2 k_{B} T_{0}^{2} \Gamma_{T}}{c_{V} V} .
$$

Comparing Eq. (A15) to Eq. (A13) then lends a calculable form of the decay rate $\Gamma_{T}$ :

$$
\Gamma_{T}=D_{T} \frac{\int d^{3} \vec{r}\left\{\vec{\nabla}\left[\epsilon(\vec{r})|\vec{E}(\vec{r})|^{2}\right]\right\}^{2}}{\int d^{3} \vec{r} \epsilon(\vec{r})^{2}|\vec{E}(\vec{r})|^{4}}
$$

Combining Eqs. (A14)-(A16) with the first-order perturbation theory relationship $\delta \omega_{0}(t) \approx-\left(\omega_{0} / n\right) \alpha_{\mathrm{TR}} \delta \bar{T}(t)$ lends the desired autocorrelation of the cavity resonance frequency

$$
\langle\delta \omega(t) \delta \omega(t+\tau)\rangle \approx \delta \omega_{\mathrm{rms}}^{2} e^{-\Gamma_{T}|\tau|},
$$

for the rms resonant frequency fluctuation

$$
\delta \omega_{\mathrm{rms}}=\frac{\omega_{0} \alpha_{\mathrm{TR}}}{n} \sqrt{\frac{k_{B} T_{0}^{2}}{c_{V} V_{T}}} .
$$

\section{Derivation of the thermal mode volume}

The same correspondence to Eq. (A1) can be used to solve for the thermal mode volume $V_{T}$ [45]. For completeness, we recapitulate this derivation. In an infinite homogeneous medium, we can solve Eq. (A7) using Fourier modes instead of introducing the phenomenological parameter $\Gamma_{T}$, yielding 


$$
\delta T(\omega, \vec{k})=\frac{F(\omega, \vec{k})}{i \omega+D_{T}|\vec{k}|^{2}}
$$

Taking the temperature mode average in Eq. (A6) and inverse Fourier transforming yields

$$
\begin{aligned}
\delta \bar{T}(t)= & \frac{1}{(2 \pi)^{4} V_{\mathrm{eff}}} \int d^{3} \vec{r} \frac{\epsilon|\vec{E}(\vec{r})|^{2}}{\max \left\{\epsilon|\vec{E}(\vec{r})|^{2}\right\}} \int d \omega e^{-i \omega t} \\
& \times \int d^{3} \vec{k} e^{i \vec{k} \cdot \vec{r}} \frac{F(\omega, \vec{k})}{i \omega+D_{T}|\vec{k}|^{2}} .
\end{aligned}
$$

Using the frequency-space autocorrelation of the Langevin driving force [compare Eq. (A13)] [122],

$$
\begin{aligned}
& \left\langle F\left(\omega_{1}, \vec{k}_{1}\right) F^{*}\left(\omega_{2}, \vec{k}_{2}\right)\right\rangle \\
& \quad=(2 \pi)^{4} \frac{2 k_{B} T_{0}^{2} D_{T}}{c_{V}}|\vec{k}|^{2} \delta\left(\vec{k}_{1}-\vec{k}_{2}\right) \delta\left(\omega_{1}-\omega_{2}\right),
\end{aligned}
$$

we can then solve for the autocorrelation of $\delta \bar{T}$ :

$$
\begin{aligned}
\left\langle\delta \bar{T}(t) \delta \bar{T}^{*}(t+\tau)\right\rangle= & \frac{\left[\max \left\{\epsilon|\vec{E}(\vec{r})|^{2}\right\}\right]^{-2}}{(2 \pi)^{3} V_{\mathrm{eff}}^{2}} \frac{k_{B} T_{0}^{2}}{c_{V}} \\
& \times\left.\left.\int d^{3} \vec{k} e^{-D_{T}|\vec{k}|^{2} \tau}\left|\int d^{3} \vec{r} \epsilon(\vec{r})\right| \vec{E}(\vec{r})\right|^{2} e^{i \vec{k} \cdot \vec{r}}\right|^{2} .
\end{aligned}
$$

Equation (A22) must equal Eq. (A1) for $\tau=0$, which reveals the final solution for the thermal mode volume $V_{T}$ :

$$
\begin{aligned}
V_{T} & =\frac{(2 \pi)^{3} V_{\mathrm{eff}}^{2}\left[\max \left\{\epsilon|\vec{E}(\vec{r})|^{2}\right\}\right]^{2}}{\left.\left.\int d^{3} \vec{k}\left|\int d^{3} \vec{r} \epsilon(\vec{r})\right| \vec{E}(\vec{r})\right|^{2} e^{i \vec{k} \cdot \vec{r}}\right|^{2}} \\
& =\frac{V_{\mathrm{eff}}^{2}\left[\max \left\{\epsilon|\vec{E}(\vec{r})|^{2}\right\}\right]^{2}}{\int d^{3} \vec{r} \epsilon(\vec{r})^{2}|\vec{E}(\vec{r})|^{4}}=\frac{V_{\mathrm{eff}}^{2}}{V_{\mathrm{eff}}^{(2)}},
\end{aligned}
$$

where

$$
V_{\mathrm{eff}}^{(2)}=\frac{\int d^{3} \vec{r} \epsilon(\vec{r})^{2}|\vec{E}(\vec{r})|^{4}}{\max \left\{\epsilon^{2}|\vec{E}|^{4}\right\}} .
$$

This result matches Gorodetsky's original result [45] with the exception of different normalization conditions.

\section{Comparison to multimode thermal decay in a 2D PhC slab}

Under the single-mode approximation derived in the preceding sections, Eq. (A17) implies a Lorentzian TRN spectrum

$$
S_{\omega \omega}(\omega)=\left(\frac{\omega_{0}}{n} \alpha_{\mathrm{TR}}\right)^{2} \frac{k_{B} T_{0}^{2}}{c_{V} V_{T}} \frac{2 \Gamma_{T}}{\Gamma_{T}^{2}+\omega^{2}} .
$$

As noted in the main text, this approximate spectrum can be evaluated for any optical microcavity (photonic crystals, microtoroids, microbottles, ring resonators, micropillars, microdisks, and so on) independent of its exact confining geometry. This feature allows us to derive general noise limits as illustrated in the main text and derived in Appendix A 4. If a particular experimental system is of interest, we can verify the accuracy of this approximation by solving the stochastic heat equation [Eq. (A7)] for that particular cavity geometry. Here, since we measure TRN in high- $Q / V_{\text {eff }} 2 \mathrm{D}$ slab photonic crystal cavities (see the main text and Appendix B), we demonstrate this evaluation for a Gaussian mode confined within an infinite twodimensional slab. The heat equation in this case lends logarithmically—as opposed to exponentially—decaying temperature fluctuations in time.

For a slab of thickness $w$ lying atop the $x y$ plane, the local temperature change $\delta T(\vec{r}, t)=\sum_{n} T_{n}\left(\vec{r}_{\|}, t\right) \phi_{n}(z)$ can be expanded in terms of the out-of-plane eigenfunctions $\phi_{n}(z)=\cos (n \pi z / w)$ assuming insulating boundary conditions on the top and bottom of the slab. The stochastic heat equation then simplifies to the form

$$
\begin{aligned}
\frac{\partial T_{n}\left(\vec{r}_{\|}, t\right)}{\partial t}= & D_{T}\left[\nabla^{2}-(n \pi / w)^{2}\right] T_{n}\left(\vec{r}_{\|}, t\right) \\
& +\frac{1}{w} \int \phi_{n}^{*}(z) F_{T}(\vec{r}, t) d z .
\end{aligned}
$$

If we assume a two-dimensional Gaussian mode profile

$$
\epsilon(\vec{r})|\vec{E}(\vec{r})|^{2}= \begin{cases}\frac{1}{2 \pi \sigma^{2}} e^{-\left|\vec{r}_{\|}\right|^{2} / 2 \sigma^{2}} & 0<z<w, \\ 0 & \text { else, }\end{cases}
$$

all $n \neq 0$ terms in the temperature expansion have zero contribution to the mode-averaged temperature fluctuation [Eq. (A6)] of interest, which involves the integral $\int \phi_{n}(z) d z$. Equation (A26) then simplifies to the twodimensional form

$$
\frac{\partial T\left(\vec{r}_{\|}, t\right)}{\partial t}=D_{T} \nabla^{2} T\left(\vec{r}_{\|}, t\right)+F_{T}^{\|}\left(\vec{r}_{\|}, t\right),
$$

where we drop the $n=0$ subscript and introduce a modified fluctuation $F_{T}^{\|}\left(\vec{r}_{\|}, t\right)$ with statistics

$$
\begin{aligned}
& \left\langle F_{T}^{\| *}\left(\vec{r}_{\|}, t\right) F_{T}^{\|}\left(\vec{r}_{\|}^{\prime}, t^{\prime}\right)\right\rangle \\
& \quad=\frac{1}{w^{2}} \int d z \int d z^{\prime} \phi_{0}^{*}(z) \phi_{0}\left(z^{\prime}\right)\left\langle F_{T}^{*}(\vec{r}, t) F_{T}\left(\vec{r}^{\prime}, t^{\prime}\right)\right\rangle \\
& \quad=\frac{1}{w} \frac{2 D_{T} k_{B} T_{0}^{2}}{c_{V}} \delta\left(t-t^{\prime}\right) \vec{\nabla}_{\vec{r}_{\|}} \cdot \vec{\nabla}_{\vec{r}_{\|}^{\prime}} \delta\left(\vec{r}_{\|}-\vec{r}_{\|}^{\prime}\right) .
\end{aligned}
$$


Comparison of single-mode and multimode TRN spectra
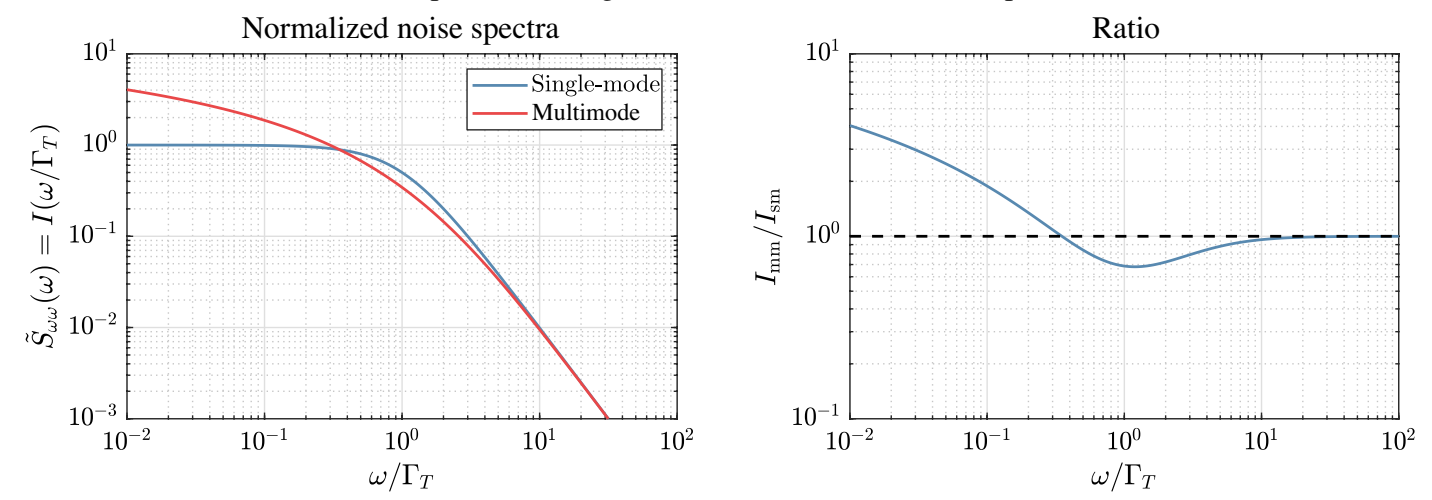

FIG. 7. Normalized noise spectra $\tilde{S}_{\omega \omega}(\omega)=I\left(\omega / \Gamma_{T}\right)$ for single-mode [Eq. (A31)] and multimode [Eq. (A30)] TRN in an infinite slab of finite thickness.

Comparing Eqs. (A28) and (A29) to their threedimensional analogs [Eqs. (A7) and (A8)], we see that projecting onto the $n=0$ subspace reduces the finitethickness slab to an infinite two-dimensional problem where $F_{T}$ is scaled by $w^{-1 / 2}$. We can then apply the techniques of Appendix A 2 (expansion in Fourier normal modes) to solve for the spectrum of temperature (and, therefore, resonant frequency) fluctuations. Without inverse Fourier transforming frequency, the autocorrelation of Eq. (A20) gives

$$
\begin{aligned}
& S_{\omega \omega}^{\mathrm{mm}}(\omega) \\
& =2\left(\frac{\omega_{0}}{n} \alpha_{\mathrm{TR}}\right)^{2} \frac{k_{B} T_{0}^{2}}{w c_{V}} \int \frac{D_{T} k_{\|}^{2}}{\left(D_{T} k_{\|}^{2}\right)^{2}+\omega^{2}}\left|\epsilon E^{2}\left(k_{\|}\right)\right|^{2} d^{2} k_{\|} \\
& =\left(\frac{\omega_{0}}{n} \alpha_{\mathrm{TR}}\right)^{2} \frac{k_{B} T_{0}^{2}}{2 \pi w c_{v} D_{T}} \underbrace{\int_{0}^{\infty} \frac{x}{x^{2}+\left(\omega \sigma^{2} / D_{T}\right)^{2}} e^{-x} d x}_{I_{\mathrm{mm}}\left(\omega \sigma^{2} / D_{T}\right)}
\end{aligned}
$$

with the change of variables $\left(k_{\|} \sigma\right)^{2} \rightarrow x$. Note that we treat the effect of patterned holes in our experimental structures through a reduced thermal conductivity, and, therefore, thermal diffusivity, as a function of the slab porosity (see Appendix B for further detail). We can compare this result with the single-mode approximation, which [by evaluating Eqs. (A16) and (A23) for the Gaussian mode profile in Eq. (A27)] gives the thermal mode volume $V_{T}=4 \pi w \sigma^{2}$, decay rate $\Gamma_{T}=D_{T} / \sigma^{2}$, and noise spectrum

$$
S_{\omega \omega}^{\mathrm{sm}}(\omega)=\left(\frac{\omega_{0}}{n} \alpha_{\mathrm{TR}}\right)^{2} \frac{k_{B} T_{0}^{2}}{2 \pi \omega c_{v} D_{T}} \underbrace{\frac{1}{1+\left(\omega \sigma^{2} / D_{T}\right)^{2}}}_{I_{\mathrm{sm}}\left(\omega \sigma^{2} / D_{T}\right)} .
$$

As expected, the integral $\int_{-\infty}^{\infty} S_{\omega \omega} d \omega / 2 \pi$ of either spectra yields $\left\langle\delta \omega^{2}\right\rangle=\left(\omega_{0} \alpha_{\mathrm{TR}} / n\right)^{2}\left\langle\delta T^{2}\right\rangle=\left(\omega_{0} \alpha_{\mathrm{TR}} / n\right)^{2} k_{B} T_{0}^{2} / c_{V} V_{T}$ in correspondence with Eq. (A1). Figure 7 plots each normalized spectrum for comparison along with the ratio $I_{\mathrm{mm}} / I_{\mathrm{sm}}$. These results substantiate the claims in the main text: the single-mode approximation undershoots at low frequency $\omega \ll \Gamma_{T}$, slightly overshoots at intermediate frequencies $\omega \sim \Gamma_{T}$, and converges to the multimode spectrum at high frequencies $\omega \gg \Gamma_{T}$. We further note that the error of the multimode spectrum increases at low frequencies for any finite volume system: in our experiment, for example, the multimode estimate does not account for lowfrequency heat transfer through the underlying oxide around the released membrane. Thus, in the range of frequencies of interest (i.e., near the thermal cutoff frequency $\Gamma_{T}=D_{T} / \sigma^{2}$ ), single-mode thermal decay is an appropriate simplifying assumption that allows the thermal noise spectrum to be well approximated irrespective of the cavity's exact geometry.

\section{Derivation of driven cavity dynamics}

To determine the practical impact of thermorefractive noise on microcavity dynamics, we now consider the case of a cavity driven by a monochromatic laser with frequency $\omega_{L}$. Intuitively, we would expect that the large (relative to the cavity linewidth), fast (relative to the cavity decay time) stochastic deviations of the resonance frequency in the high- $Q / V_{\text {eff }}$ limit would restrict the maximum intensity in the cavity, as a narrow linewidth laser would no longer always be on resonance with the fluctuating cavity resonance. A mode-volume-dependent maximum "effective" quality factor describing the stored energy should result. A similar effective quality factor could be derived if the coherence of the intracavity field-rather than the stored energy alone-is also of interest.

To prove these suppositions, we solve the driven temporal coupled mode theory relation

$$
\frac{d a(t)}{d t}=\left[i \omega_{0}(t)-\Gamma_{l}\right] a(t)+\sqrt{2 \Gamma_{c}} s_{\mathrm{in}}(t),
$$


for the cavity field $a(t)$, where $\omega_{0}(t)=\omega_{0}+\delta \omega(t)$ is the instantaneous resonant frequency, $\Gamma_{l}=\omega_{0} / 2 Q_{l}$ is the amplitude decay rate of $a$ corresponding to a loaded quality factor $Q_{l}$, and $\Gamma_{c}$ is the amplitude coupling rate of the drive field $s_{\text {in }}(t)=\tilde{s}_{\text {in }} e^{i \omega_{L} t}+$ c.c. detuned from resonance by $\Delta=\omega_{L}-\omega_{0}$. In the presence of TRN, $\delta \omega(t)$ is nonMarkovian, zero-mean Gaussian noise with the autocorrelation given by Eq. (A17). Solving with an integrating factor and introducing the slowly varying cavity amplitude $\tilde{a}(t)=a(t) e^{-i \omega_{L} t}$ in a reference frame corotating with the drive frequency $\omega_{L}$, we find

$\tilde{a}(t)=\sqrt{2 \Gamma_{c}} \tilde{s}_{\mathrm{in}} \int_{-\infty}^{t} d t^{\prime} e^{-\left(i \Delta+\Gamma_{l}\right)\left(t-t^{\prime}\right)} e^{i \int_{t^{\prime}}^{t} d t^{\prime \prime} \delta \omega\left(t^{\prime \prime}\right)}$.

Since the steady-state solution is desired, we assume that the integration starts at $t=-\infty$ such that the system has no "memory" of the initial conditions. Using Eq. (A33), we can compute $\langle\tilde{a}(t)\rangle$ and $\left\langle|\tilde{a}(t)|^{2}\right\rangle$, the mean cavity field amplitude and stored energy, respectively. In certain limiting cases, the noise spectrum $S_{a a}(\omega)$ of the intracavity field can also be derived.

\section{a. Cavity spectrum in the perturbative limit}

One of these limiting cases is the perturbative regime commonly studied in the literature for low- $Q / V_{\text {eff }}$ cavities, wherein $\delta \omega_{\text {rms }} \ll \Gamma_{l}$. In this case, $\tilde{a}(t)$ and $\delta \omega(t)-$ described by [cf. Eq. (A9)]

$$
\frac{d \delta \omega(t)}{d t}=-\Gamma_{T} \delta \omega(t)+\delta \omega_{\mathrm{rms}} \sqrt{2 \Gamma_{T}} W(t)
$$

for a Wiener process $W(t)$ with $\left\langle W(t) W\left(t^{\prime}\right)\right\rangle=\delta\left(t-t^{\prime}\right)$ can be expanded in orders of $\delta \omega_{\text {rms }} \sqrt{2 \Gamma_{T}}$. The zeroth- and first-order evolution equations (with subscripts 0 and 1 , respectively) are

$$
\begin{gathered}
\frac{d \tilde{a}_{0}(t)}{d t}=\left\{i\left[\delta \omega_{0}(t)-\Delta\right]-\Gamma_{l}\right\} \tilde{a}_{0}(t)+\sqrt{2 \Gamma_{c}} \tilde{s}_{\text {in }} \\
\frac{d \tilde{a}_{1}(t)}{d t}=\left\{i\left[\delta \omega_{0}(t)-\Delta\right]-\Gamma_{l}\right\} a_{1}(t)+i \delta \omega_{1}(t) \tilde{a}_{0}(t), \\
\frac{d \delta \omega_{0}(t)}{d t}=-\Gamma_{T} \delta \omega_{0}(t)
\end{gathered}
$$

$$
\frac{d \delta \omega_{1}(t)}{d t}=-\Gamma_{T} \delta \omega_{1}(t)+\delta \omega_{\mathrm{rms}} \sqrt{2 \Gamma_{T}} W(t) .
$$

Solving in the frequency domain yields

$$
\tilde{a}_{1}(\omega)=\frac{\sqrt{2 \Gamma_{c}} \tilde{s}_{\mathrm{in}}}{\Gamma_{l}+i \Delta} \frac{i \delta \omega_{\mathrm{rms}} \sqrt{2 \Gamma_{T}} W(\omega)}{\left(\Gamma_{T}-i \omega\right)\left[\Gamma_{l}+i(\omega+\Delta)\right]},
$$

corresponding to the frequency spectrum
$S_{a a}(\omega)=\frac{2 \Gamma_{c}\left|\tilde{s}_{\mathrm{in}}\right|^{2}}{\Gamma_{l}^{2}+\Delta^{2}} \frac{2 \Gamma_{T} \delta \omega_{\mathrm{rms}}^{2}}{\left(\Gamma_{T}^{2}+\omega^{2}\right)\left(\Gamma_{l}^{2}+(\omega+\Delta)^{2}\right)}$.

The intracavity noise spectrum can therefore be approximated as the product of two Lorentzians with spectral widths $2 \Gamma_{T}$ and $2 \Gamma_{l}$. When $\Gamma_{T} \ll \Gamma_{l}$, which often coincides with the perturbative limit $\delta \omega_{\text {rms }} \ll \Gamma_{l}$ for large mode volumes $\left(\delta \omega_{\mathrm{rms}} \propto V_{T}^{-1 / 2}\right.$ and $\Gamma_{T} \propto V_{T}^{-2 / 3}$ for a three-dimensional Gaussian mode), the resonant frequency fluctuations are small and occur over timescales much longer than that of intracavity photon decay. Thus, TRN leads to a weak inhomogeneous broadening of the resonant mode that can often be neglected for common applications of low- $Q / V_{\text {eff }}$ optical cavities. Gravitational wave interferometry [6,122] and ultrastable optical frequency references $[47,123]$ are two notable exceptions that have led to significant interest in perturbative TRN.

\section{b. General derivation for $\langle\tilde{\boldsymbol{a}}(\boldsymbol{t})\rangle$}

Our work focuses on the transition to nonperturbative TRN in high- $Q / V_{\text {eff }}$ microcavities, where we are interested in general solutions for the statistical moments of Eq. (A33) in the presence of TRN. Specifically, $\langle\tilde{a}(t)\rangle$ provides insight into thermal-noise-induced dephasing while $\left\langle\tilde{a}^{2}(t)\right\rangle$ lends a bound on the maximum allowable energy storage.

The expected intracavity field amplitude

$$
\langle\tilde{a}(t)\rangle=\sqrt{2 \Gamma_{c}} \tilde{s}_{\text {in }} \int_{-\infty}^{t} d t^{\prime} e^{-\left(i \Delta+\Gamma_{l}\right)\left(t-t^{\prime}\right)}\left\langle e^{\int_{t^{\prime}}^{t} d t^{\prime \prime} i \delta \omega\left(t^{\prime \prime}\right)}\right\rangle
$$

follows directly from Eq. (A33), where the average on the right-hand side has a similar form to the characteristic functional [60]

$\Phi[k(t)]=\left\langle e^{i \int k(t) f(t) d t}\right\rangle=\frac{\int e^{i \int k(t) f(t) d t} P[f(t)] \mathcal{D} f(t)}{\int P[f(t)] \mathcal{D} f(t)}$,

a normalized average of $e^{i \int k(t) f(t) d t}$ along the paths $f(t)$ with respective probabilities $P[f(t)]$. For the special case of Gaussian noise, the moment-generating properties of the characteristic functional allow Eq. (A42) to be simplified to

$\Phi[k(t)]=e^{i \int k(t) M(t) d t} e^{-1 / 2 \int d t \int d t^{\prime} k(t) k\left(t^{\prime}\right)\left\langle f(t) f\left(t^{\prime}\right)\right\rangle}$,

which is characterized by two parameters only: the mean path $M(t)$ and the autocorrelation of the noise $f(t)$, $\left\langle f(t) f\left(t+t^{\prime}\right)\right\rangle$. Comparing Eq. (A41) to Eq. (A42), we find

$$
k\left(t^{\prime \prime}\right)= \begin{cases}1 & t^{\prime}<t^{\prime \prime}<t \\ 0 & \text { else }\end{cases}
$$


and $f(t)=\delta \omega\left(t^{\prime \prime}\right)$. Since $\left\langle\omega_{0}(t)\right\rangle=0$,

$$
\begin{aligned}
\Phi & =\exp \left[-\frac{1}{2} \int_{t^{\prime}}^{t} d t_{2}^{\prime} \int_{t^{\prime}}^{t} d t_{2}^{\prime \prime} \delta \omega_{\mathrm{rms}}^{2} e^{-\Gamma_{T}\left|t_{2}^{\prime \prime}-t_{2}^{\prime}\right|}\right] \\
& =\exp \left[\frac{\delta \omega_{\mathrm{rms}}^{2}}{\Gamma_{T}^{2}}\left[1-\Gamma_{T}\left(t-t^{\prime}\right)-e^{-\Gamma_{T}\left(t-t^{\prime}\right)}\right]\right]
\end{aligned}
$$

The above form, combined with the change of variable $\tilde{\tau}=\left(\delta \omega_{\mathrm{rms}} / \Gamma_{T}\right)^{2} e^{-\Gamma_{T}\left(t-t^{\prime}\right)}$, simplifies to

$$
\begin{aligned}
\langle\tilde{a}(t)\rangle= & \frac{\sqrt{2 \Gamma_{c}} \tilde{s}_{\mathrm{in}}}{\Gamma_{T}} e^{\left(\delta \omega_{\mathrm{rms}} / \Gamma_{T}\right)^{2}}\left(\frac{\delta \omega_{\mathrm{rms}}}{\Gamma_{T}}\right)^{2\left\{\left[-i \Delta-\Gamma_{l}-\left(\delta \omega_{\mathrm{rms}}^{2} / \Gamma_{T}\right)\right] / \Gamma_{T}\right\}} \\
& \times \int_{0}^{\left(\delta \omega_{\mathrm{rms}} / \Gamma_{T}\right)^{2}} \tilde{\tau}^{\left\{\left[i \Delta+\Gamma_{l}+\left(\delta \omega_{\mathrm{rms}}^{2} \Gamma_{T}\right)\right] / \Gamma_{T}\right\}-1} e^{-\tilde{\tau}} d \tilde{\tau}
\end{aligned}
$$

which is in the form of the lower incomplete Gamma function

$$
\gamma_{l}(s, x)=\int_{0}^{x} \tilde{\tau}^{s-1} e^{-\tilde{\tau}} d \tilde{\tau} .
$$

The final closed-form solution is, therefore,

$$
\begin{aligned}
\langle\tilde{a}(t)\rangle & =\frac{\sqrt{2 \Gamma_{c}} \tilde{s}_{\text {in }}}{\Gamma_{T}} e^{x} x^{-s} \gamma_{l}(s, x), \\
x & \equiv\left(\frac{\delta \omega_{\mathrm{rms}}}{\Gamma_{T}}\right)^{2} \\
s & \equiv \frac{\Gamma_{l}+i \Delta}{\Gamma_{T}}+x
\end{aligned}
$$

To confirm this solution, we can evaluate the limiting case of $\delta \omega_{\mathrm{rms}} \rightarrow 0(x \rightarrow 0)$, corresponding to a noiseless thermal reservoir when $T \rightarrow 0$. Using the series expansion of $\gamma_{l}(s, x)$ in terms of $s, x$, and the complete Gamma function $\gamma_{f}(z)$, we find

$$
\begin{aligned}
\langle\tilde{a}(t)\rangle_{T=0} & =\frac{\sqrt{2 \Gamma_{c}} \tilde{s}_{\text {in }}}{\Gamma_{T}} \frac{\gamma_{f}(s)}{\gamma_{f}(s+1)}=\left.\frac{\sqrt{2 \Gamma_{c}} \tilde{s}_{\text {in }}}{\Gamma_{T}} \frac{1}{s}\right|_{x=0} \\
& =\frac{\sqrt{2 \Gamma_{c}} \tilde{s}_{\text {in }}}{\Gamma_{l}+i \Delta}
\end{aligned}
$$

as expected from noiseless temporal coupled mode theory. Assuming critical coupling $\left(\Gamma_{c}=\Gamma_{l} / 2\right)$ and resonant excitation $(\Delta=0)$, we find the "effective" quality factor

$$
Q_{\text {eff }}=\frac{\omega_{0}|\langle\tilde{a}(t)\rangle|^{2}}{2\left|\tilde{s}_{\text {in }}\right|^{2}}=Q_{l}\left(\frac{\Gamma_{l}}{\Gamma_{T}}\right)^{2} e^{2 x} x^{-2 s} \gamma_{l}^{2}(s, x)
$$

by analogy to the noiseless result where $Q=\omega_{0}|\langle\tilde{a}(t)\rangle|_{T=0}^{2} /$ $2\left|\tilde{s}_{\text {in }}\right|^{2}$.

This result is used in the main text to describe dephasing in the qubit limit of cavity nonlinear optics. For a given mode volume, the optimum loaded quality factor $Q_{l}^{\mathrm{opt}} \approx$ $\omega_{0} \Gamma_{T} / 2 \delta \omega_{\mathrm{rms}}^{2}$ (assuming $\delta \omega_{\mathrm{rms}} \ll \Gamma_{T}$, which is valid for the range of mode volumes plotted in Fig. 5 of the main text) maximizes the resonant cavity amplitude: lower quality factors incur excess loss, whereas higher quality factors allow the qubit to "explore" a larger region of the phase space, thereby reducing the integrated cavity amplitude. Intuitively, the resulting maximum amplitude $|\langle\tilde{a}(t)\rangle|$ increases with increasing mode volume due to the reduced magnitude of temperature fluctuations.

\section{c. General derivation for $\left\langle\tilde{\boldsymbol{a}}^{\mathbf{2}}(\boldsymbol{t})\right\rangle$}

Solving for $\left\langle\tilde{a}(t)^{2}\right\rangle$ generally follows the same procedure and reveals a limit on the allowable intracavity optical energy in the presence of TRN. Starting from Eq. (A33), the amplitude correlation takes the form

$$
\begin{aligned}
\left\langle\tilde{a}(t) \tilde{a}^{*}(0)\right\rangle= & 2 \Gamma_{c}\left|\tilde{s}_{\text {in }}\right|^{2} \int_{-\infty}^{t} d t^{\prime} e^{-\left(i \Delta+\Gamma_{l}\right)\left(t-t^{\prime}\right)} \\
& \times \int_{-\infty}^{0} d t^{\prime \prime} e^{-\left(i \Delta-\Gamma_{l}\right) t^{\prime \prime}}\left\langle e^{\int_{t^{\prime}}^{t} i \delta \omega\left(t_{2}\right) d t_{2}-\int_{t^{\prime \prime}}^{0} i \delta \omega\left(t_{2}\right) d t_{2}}\right\rangle .
\end{aligned}
$$

Following the method of Ref. [59], the average can be expressed in the form of Eq. (A42):

$$
\left\langle e^{\int_{t^{\prime}}^{t} i \delta \omega\left(t_{2}\right) d t_{2}-\int_{t^{\prime \prime}}^{0} i \delta \omega\left(t_{2}\right) d t_{2}}\right\rangle=\left\langle e^{i \int_{-\infty}^{\infty} k\left(t_{2}\right) \delta \omega\left(t_{2}\right) d t_{2}}\right\rangle,
$$

by appropriately defining $k(t)$. As illustrated in Fig. 8, the dependence of $k\left(t_{2}\right)$ upon $t^{\prime}, t^{\prime \prime}$, and $t$ differs in three sectors of the region of integration. Simple diagrams in each of the three scenarios can be used to find a closed form for $k(t)$ :

$$
k\left(t_{2}\right)= \begin{cases}\operatorname{sgn}\left(t^{\prime \prime}-t^{\prime}\right), & t^{\prime}<0 \text { and } \min \left(t^{\prime}, t^{\prime \prime}\right) \leq t_{2} \leq \max \left(t^{\prime}, t^{\prime \prime}\right) \\ 1, & \left(t^{\prime}<0 \text { and } 0 \leq t_{2} \leq t\right) \text { or }\left(t^{\prime}>0 \text { and } t^{\prime} \leq t_{2} \leq t\right) \\ -1, & t^{\prime}>0 \text { and } t^{\prime \prime} \leq t_{2} \leq 0 \\ 0, & \text { else. }\end{cases}
$$

This definition allows us to rewrite the autocorrelation as 


$$
\begin{aligned}
\mathcal{R}_{a a}(t) & =\left\langle\tilde{a}(t) \tilde{a}^{*}(0)\right\rangle^{*} \\
& =2 \Gamma_{c}\left|\tilde{s}_{\mathrm{in}}\right|^{2} \int_{-\infty}^{t} d t^{\prime} e^{\left(i \Delta-\Gamma_{l}\right)\left(t-t^{\prime}\right)} \int_{-\infty}^{0} d t^{\prime \prime} e^{\left(i \Delta+\Gamma_{l}\right) t^{\prime \prime}} \exp \left[-\frac{1}{2} \int_{-\infty}^{\infty} d t_{2}^{\prime} \int_{-\infty}^{\infty} d t_{2}^{\prime \prime} k\left(t_{2}^{\prime}\right) k\left(t_{2}^{\prime \prime}\right) \delta \omega_{\mathrm{rms}}^{2} e^{-\Gamma_{T}\left|t_{2}^{\prime}-t_{2}^{\prime \prime}\right|}\right] .
\end{aligned}
$$

While a general solution to the full autocorrelation in Eq. (A55) appears intractable, we can find $\left\langle|\tilde{a}|^{2}\right\rangle=$ $\left\langle|\tilde{a}(0)|^{2}\right\rangle$ by evaluating Eq. (A55) at $t=0$ (thereby eliminating integration region 3 in Fig. 8), which yields

$$
\begin{aligned}
\left\langle|\tilde{a}|^{2}\right\rangle= & 2 \Gamma_{c}\left|\tilde{s}_{\text {in }}\right|^{2} \int_{-\infty}^{0} d t^{\prime} e^{\left(i \Delta+\Gamma_{l}\right) t^{\prime}} \int_{-\infty}^{0} d t^{\prime \prime} e^{\left(-i \Delta+\Gamma_{l}\right) t^{\prime \prime}} \\
& \times \exp \left[\left(\frac{\delta \omega_{\mathrm{rms}}}{\Gamma_{T}}\right)^{2}\left(1-\Gamma_{T}\left|t^{\prime \prime}-t^{\prime}\right|-e^{-\Gamma_{T}\left|t^{\prime \prime}-t^{\prime}\right|}\right)\right] .
\end{aligned}
$$

Note that the integral in region 1 or region 2 is nearly the same-exchanging $t^{\prime}$ and $t^{\prime \prime}$ in either region returns the integral for the other region but conjugates $i \Delta$. Therefore, we focus on evaluating Eq. (A56) in region $1\left(t^{\prime \prime}>t^{\prime}\right)$ and then generalize this result to the other region by taking the complex conjugate. In region 1 , the substitution $\tilde{\tau}=$ $\left(\delta \omega_{\text {rms }} / \Gamma_{T}\right)^{2} \exp \left[-\Gamma_{T}\left(t^{\prime \prime}-t^{\prime}\right)\right]$ yields

$$
\begin{aligned}
\left\langle|\tilde{a}(0)|^{2}\right\rangle_{1}= & 2 \Gamma_{c}\left|\tilde{s}_{\text {in }}\right|^{2} \int_{-\infty}^{0} d t^{\prime} e^{2 \Gamma_{l} t^{\prime}} \frac{e^{x}}{\Gamma_{T}} x^{-s^{\prime}} \int_{x e^{\Gamma_{T} t^{\prime}}}^{x} \tilde{\tau}^{s^{\prime}-1} e^{-\tilde{\tau}} d \tilde{\tau} \\
= & 2 \Gamma_{c}\left|\tilde{s}_{\text {in }}\right|^{2} \frac{e^{x}}{\Gamma_{T}} x^{-s^{\prime}} \\
& \times \int_{-\infty}^{0} d t^{\prime} e^{2 \Gamma_{l} t^{\prime}}\left[\gamma_{l}\left(s^{\prime}, x\right)-\gamma_{l}\left(s^{\prime}, x e^{\Gamma_{T} t^{\prime}}\right)\right], \quad \text { (A57) }
\end{aligned}
$$

where $x=\left(\delta \omega_{\mathrm{rms}} / \Gamma_{T}\right)^{2}$ and $s^{\prime}=\left(\delta \omega_{\mathrm{rms}}^{2} / \Gamma_{T}-\Gamma_{l}+i \Delta\right) / \Gamma_{T}$. The first term can be directly evaluated, while the second can be simplified with integration by parts using the relationship

$$
\frac{\partial \gamma_{l}\left(s^{\prime}, x\right)}{\partial x}=x^{s^{\prime}-1} e^{-x}
$$

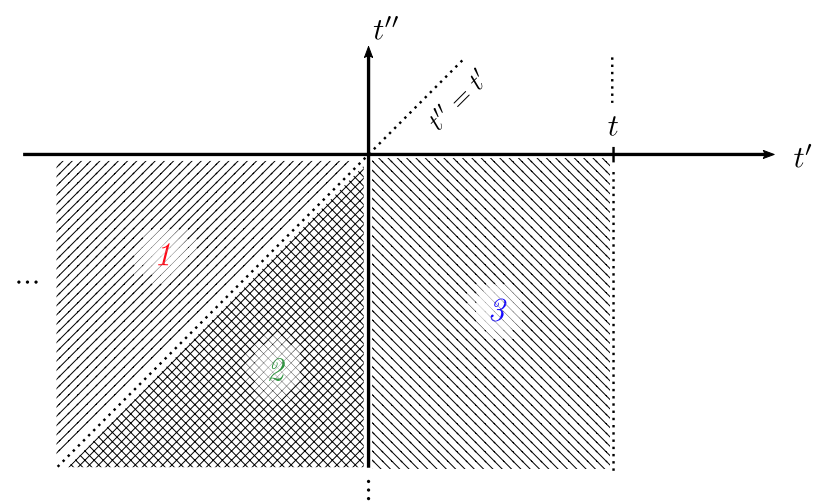

FIG. 8. The complete region of integration can be divided into three subspaces which yield different conditions for $k(t)$.
With a second substitution $\tilde{\tau}_{2}=x e^{\Gamma_{T} t^{\prime}}$, we find

$$
\begin{aligned}
\left\langle|\tilde{a}(0)|^{2}\right\rangle_{1}= & 2 \Gamma_{c}\left|\tilde{s}_{\mathrm{in}}\right|^{2} \frac{e^{x}}{\Gamma_{T}} x^{-s^{\prime}}\left[\frac{\gamma_{l}\left(s^{\prime}, x\right)}{2 \Gamma_{l}}\right. \\
& \left.-\frac{x^{-2 \Gamma_{l} / \Gamma_{T}}}{\Gamma_{T}} \int_{0}^{x} \tilde{\tau}_{2}^{\left(2 \Gamma_{l} / \Gamma_{T}\right)-1} \gamma_{l}\left(s^{\prime}, \tilde{\tau}_{2}\right) d \tilde{\tau}_{2}\right] \\
= & 2 \Gamma_{c}\left|\tilde{s}_{\mathrm{in}}\right|^{2} \frac{e^{x}}{\Gamma_{T}} x^{-s^{\prime}}\left\{\frac{\gamma_{l}\left(s^{\prime}, x\right)}{2 \Gamma_{l}}-\frac{x^{-2 \Gamma_{l} / \Gamma_{T}}}{\Gamma_{T}}\left(\frac{\Gamma_{T}}{2 \Gamma_{l}}\right)\right. \\
& \left.\times\left[\tilde{\tau}_{2}^{2 \Gamma_{l} / \Gamma_{T}} \gamma_{l}\left(s^{\prime}, \tilde{\tau}_{2}\right)+\gamma_{u}\left(s^{\prime}+\frac{2 \Gamma_{l}}{\Gamma_{T}}, \tilde{\tau}_{2}\right)\right]_{\tilde{\tau}_{2}=0}^{\tilde{\tau}_{2}=x}\right\},
\end{aligned}
$$

where $\gamma_{u}\left(s^{\prime}, x\right)$ is the upper incomplete Gamma function defined by

$$
\gamma_{u}\left(s^{\prime}, x\right)=\int_{x}^{\infty} \tau^{s^{\prime}-1} e^{-\tau} d \tau
$$

Evaluating the final terms, the result simplifies nicely to

$$
\left\langle|\tilde{a}(0)|^{2}\right\rangle_{1}=\frac{\left|\tilde{s}_{\mathrm{in}}\right|^{2}}{\Gamma_{T}}\left(\frac{\Gamma_{c}}{\Gamma_{l}}\right) e^{x} x^{-\left[s^{\prime}+\left(2 \Gamma_{l} / \Gamma_{T}\right)\right.} \gamma_{l}\left(s^{\prime}+\frac{2 \Gamma_{l}}{\Gamma_{T}}, x\right) .
$$

To find the complete result, we simply add the second term in Eq. (A56) to find

$$
\begin{gathered}
\left\langle|\tilde{a}|^{2}\right\rangle=\frac{\left|\tilde{s}_{\text {in }}\right|^{2}}{\Gamma_{T}}\left(\frac{\Gamma_{c}}{\Gamma_{l}}\right) e^{x}\left[x^{-s} \gamma_{l}(s, x)+x^{-s^{*}} \gamma_{l}\left(s^{*}, x\right)\right], \\
x \equiv\left(\frac{\delta \omega_{\text {rms }}}{\Gamma_{T}}\right)^{2}, \\
s \equiv \frac{\Gamma_{l}+i \Delta}{\Gamma_{T}}+x .
\end{gathered}
$$

Note the similarity to Eqs. (A47)-(A49). Once again, we must ensure that our solution corresponds to the noiseless result expected when $\delta \omega_{\text {rms }} \rightarrow 0$. Using the series expansion of $\gamma_{l}(s, x)$, we find 


$$
\begin{aligned}
\left\langle|\tilde{a}|^{2}\right\rangle_{T=0} & =\frac{\left|\tilde{s}_{\text {in }}\right|^{2}}{\Gamma_{T}}\left(\frac{\Gamma_{c}}{\Gamma_{l}}\right)\left[\frac{\gamma_{f}\left(\left.s\right|_{x=0}\right)}{\gamma_{f}\left(\left.s\right|_{x=0}+1\right)}+\frac{\gamma_{f}\left(\left.s^{*}\right|_{x=0}\right)}{\gamma_{f}\left(\left.s^{*}\right|_{x=0}+1\right)}\right] \\
& =\frac{\left|\tilde{s}_{\text {in }}\right|^{2}}{\Gamma_{T}}\left(\frac{\Gamma_{c}}{\Gamma_{l}}\right)\left[\frac{\Gamma_{T}}{\Gamma_{l}+i \Delta}+\frac{\Gamma_{T}}{\Gamma_{l}-i \Delta}\right], \\
\left\langle|\tilde{a}|^{2}\right\rangle_{T=0} & =\frac{2 \Gamma_{c}\left|\tilde{s}_{\text {in }}\right|^{2}}{\Delta^{2}+\Gamma_{l}^{2}}
\end{aligned}
$$

as expected. Similar to the solution for $\langle\tilde{a}(t)\rangle$, we define the effective quality factor

$$
Q_{\mathrm{eff}}=\frac{\omega_{0}\left\langle|\tilde{a}|^{2}\right\rangle}{2\left|\tilde{s}_{\mathrm{in}}\right|^{2}}=\frac{\omega_{0}}{2 \Gamma_{T}} e^{x} x^{-s} \gamma_{l}(s, x)
$$

for resonant excitation $(\Delta=0)$ and critical coupling $\left(\Gamma_{c}=\Gamma_{l} / 2\right)$.

As opposed to the nonmonotonic scaling of the mean field amplitude $|\langle\tilde{a}(t)\rangle|$ with $Q_{l}$, the stored energy $\left\langle|\tilde{a}(t)|^{2}\right\rangle$ increases monotonically with increasing $Q_{l}$. This behavior is intuitively described in the main text: continuing to increase $Q_{l}$ decreases the cavity linewidth until $Q_{\text {eff }}$ is saturated by mode-volume-dependent thermal noise in the high- $Q_{l} / V_{\text {eff }}$ regime. Finally, we note that the maximum energy storage (although not necessarily the maximum intensity, which also depends on the mode volume) is achieved with large mode volumes due to reduced thermooptic noise.

\section{d. Cavity spectrum in the white noise limit}

The complete field autocorrelation in Eq. (A55) simplifies considerably in the high- $Q_{l}$ limit where $\Gamma_{T} \gg \Gamma_{l}$, as the cavity resonant frequency $\omega_{0}(t)$ can be assumed to directly track the temperature noise over the relevant timescales. The frequency noise is then effectively delta correlated in time, and the aforementioned-albeit tedious- "integration by regions" technique can then be similarly applied to solve for the field noise spectrum $S_{a a}(\omega)$. A more intuitive approach to this solution is through adiabatic elimination of $\omega_{0}(t)$ 's dynamics following the procedure in Ref. [124]. Converting the optical field and resonant frequency evolution equations [Eqs. (A32) and (A34)] into stochastic differential equations yields

$$
\begin{gathered}
d \tilde{a}(t)=\left(\left\{i[\delta \omega(t)-\Delta]-\Gamma_{l}\right\} \tilde{a}(t)+\sqrt{2 \Gamma_{c}} \tilde{s}_{\mathrm{in}}\right) d t, \\
d \delta \omega(t)=-\Gamma_{T} \delta \omega(t) d t+\delta \omega_{\mathrm{rms}} \sqrt{2 \Gamma_{T}} d W(t)
\end{gathered}
$$

for both Itô and Stratonovich forms, since the frequency noise is additive $\left(\delta \omega_{\mathrm{rms}} \sqrt{2 \Gamma_{T}}\right.$ is constant). In the limit $\Gamma_{T} \rightarrow \infty$, we can adiabatically eliminate the resonant frequency dynamics, yielding a steady-state value $\delta \omega(t)=$ $\sqrt{2 / \Gamma_{T}} \delta \omega_{\mathrm{rms}} d W(t) / d t$. The cavity evolution can then be simplified to

$$
\begin{aligned}
d \tilde{a}_{S}(t)= & {\left[\left(-i \Delta-\Gamma_{l}\right) \tilde{a}_{S}(t)+\sqrt{2 \Gamma_{c}} \tilde{s}_{\mathrm{in}}\right] d t } \\
& +\sqrt{\frac{2}{\Gamma_{T}} \delta \omega_{\mathrm{rms}} \tilde{a}_{S}(t) d W(t),} \\
d \tilde{a}_{I}(t)= & \left\{\left[-i \Delta-\left(\Gamma_{l}+\frac{\delta \omega_{\mathrm{rms}}^{2}}{\Gamma_{T}}\right)\right] \tilde{a}_{I}(t)+\sqrt{2 \Gamma_{c}} \tilde{s}_{\mathrm{in}}\right\} d t \\
& +\sqrt{\frac{2}{\Gamma_{T}} \delta \omega_{\mathrm{rms}} \tilde{a}_{I}(t) d W(t)}
\end{aligned}
$$

in Stratonovich and Itô forms, respectively. Applying the Itô rule $[d W(t)]^{2}=d t$ to the latter, we can solve for the steady-state moments

$$
\begin{gathered}
\langle\tilde{a}(t)\rangle=\frac{\sqrt{2 \Gamma_{c}} \tilde{s}_{\mathrm{in}}}{\left[\Gamma_{l}+\delta \omega_{\mathrm{rms}}^{2} / \Gamma_{T}\right]+i \Delta}, \\
\left\langle|\tilde{a}(t)|^{2}\right\rangle=\frac{2 \Gamma_{c}\left(1+\delta \omega_{\mathrm{rms}}^{2} / \Gamma_{T} \Gamma_{l}\right)\left|\tilde{s}_{\mathrm{in}}\right|^{2}}{\left[\Gamma_{l}+\delta \omega_{\mathrm{rms}}^{2} / \Gamma_{T}\right]^{2}+\Delta^{2}},
\end{gathered}
$$

which by comparison to Eqs. (A50) and (A65) immediately reveals a thermal broadening $2 \Gamma_{l} \rightarrow 2 \Gamma_{l}+2 \delta \omega_{\mathrm{rms}}^{2} / \Gamma_{T}$ of the microcavity linewidth. We can also derive an equation of motion for the autocorrelation $R_{a a}(\tau)=\left\langle\tilde{a}(t) \tilde{a}^{*}(t+\tau)\right\rangle$ :

$\frac{d}{d \tau} R_{a a}(\tau)=\left[i \Delta-\left(\Gamma_{l}+\frac{\delta \omega_{\mathrm{rms}}^{2}}{\Gamma_{T}}\right)\right] R_{a a}(\tau)+\sqrt{2 \Gamma_{c}} \tilde{s}_{\mathrm{in}}\langle\tilde{a}(t)\rangle$.

Solving Eq. (A73) subject to the $\tau=0$ conditions of Eq. (A72), we find

$$
\begin{aligned}
R_{a a}(\tau)= & \frac{2 \Gamma_{c}\left|s_{\mathrm{in}}\right|^{2}}{\left[\Gamma_{l}+\delta \omega_{\mathrm{rms}}^{2} / \Gamma_{T}\right]^{2}+\Delta^{2}} \\
& \times\left[\frac{\delta \omega_{\mathrm{rms}}^{2}}{\Gamma_{T} \Gamma_{l}} e^{\left(i \Delta-\Gamma_{l}-\delta \omega_{\mathrm{rms}}^{2} / \Gamma_{T}\right) \tau}+1\right],
\end{aligned}
$$

corresponding to the optical noise spectrum

$$
\begin{aligned}
S_{a a}(\omega)= & \frac{\Gamma_{c}}{\Gamma_{l}} \frac{2 \delta \omega_{\mathrm{rms}}^{2}\left|s_{\mathrm{in}}\right|^{2} / \Gamma_{T}}{\left[\Gamma_{l}+\delta \omega_{\mathrm{rms}}^{2} / \Gamma_{T}\right]^{2}+\Delta^{2}} \\
& \times \frac{2\left(\Gamma_{l}+\delta \omega_{\mathrm{rms}}^{2} / \Gamma_{T}\right)}{\left(\Gamma_{l}+\delta \omega_{\mathrm{rms}}^{2} / \Gamma_{T}\right)^{2}+(\omega+\Delta)^{2}} .
\end{aligned}
$$

Equation (A75) evaluated in the perturbative limit $\delta \omega_{\text {rms }} \ll$ $\Gamma_{l}$ coincides with the low-frequency $\left(\omega \ll \Gamma_{T}\right)$ limit of the previous perturbative spectrum [Eq. (A40)]. 


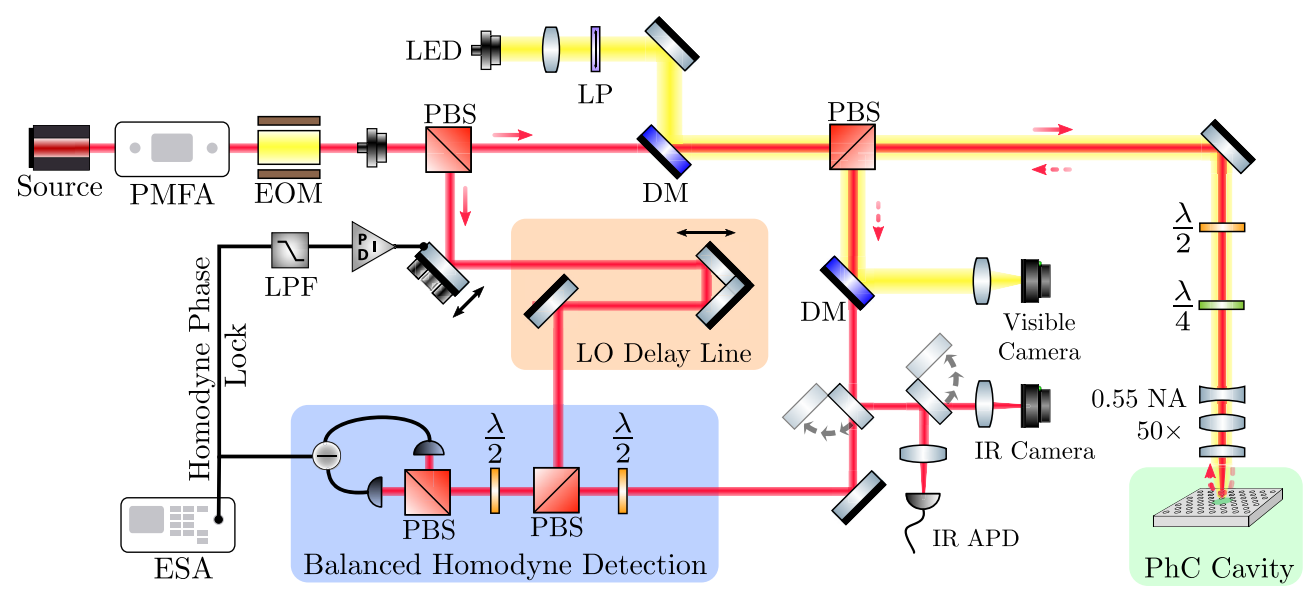

FIG. 9. Schematic of the setup built to measure TRN in photonic crystal cavities. An amplified (PriTel PMFA) continuous wave laser (Santec TSL-710) is separated into a local oscillator and cavity signal by a polarizing beam splitter (PBS). The LO line is passively pathlength matched to the cavity signal using a tunable retroreflector delay line. The cavity signal is combined with a linearly polarized (LP) white light source (LED) using a dichroic mirror (DM) and is reflected from a $\mathrm{PhC}$ cavity rotated $45^{\circ}$ from the incident polarization (adjustable with a half-wave plate, $\lambda / 2$ ), which allows the cavity signal to be isolated from the specular reflection using a PBS. A quarter-wave plate $(\lambda / 4)$ allows the specular reflection to be extracted for comparison to the cavity-only reflection. The reflected illumination light is separated and imaged onto a silicon CCD. The cavity signal can be directed with flip mirrors toward an IR camera for imaging, an IR avalanche photodetector (ThorLabs PDB410C $10 \mathrm{MHz}$ InGaAs APD) to collect low-noise reflection spectra, or toward the balanced homodyne detector. For the latter, a balanced photodetector (ThorLabs PDB480C-AC 1.6 GHz InGaAs $p$ - $i$ - $n$ photodetector) measures the homodyne signal from the recombined cavity reflection and local oscillator, and the result is recorded on an electronic spectrum analyzer (ESA; Agilent N9010A EXA Signal Analyzer). The dc signal extracted from a low-pass filter (LPF) is used as the feedback signal for a digital PID controller which stabilizes the signal-LO phase difference by actuating a piezoactuated mirror. An EOM provides a known phase noise which can be used to calibrate the frequency noise of the PhC cavity. The sample stage is temperature stabilized to $\Delta T<0.01 \mathrm{~K}$ using a Peltier plate and a feedback temperature controller.

\section{APPENDIX B: EXPERIMENTAL TRN IN PHOTONIC CRYSTAL CAVITIES}

The single-mode thermal decay approximation made in Eq. (A9) implies the decay rate of Eq. (A16) and the spectral density of cavity resonant frequency in Eq. (A25). This result is commonly used as a simplifying assumption for temperature fluctuations [35,61]; however, it is not immediately clear that the single-mode approximation holds in the case of small-mode-volume optical microcavities, where the characteristic length scales of the near diffraction-limited optical mode $(\sim \lambda / n)$ can approach that of the phonon mean free path [75]. In the absence of any experimental data in the literature to verify the assumption, we construct an experiment to measure thermorefractive noise in high- $Q_{l} / V_{\text {eff }}$ silicon photonic crystal cavities. The experiment also allows us to compare measured TRN with the spectra derived from our multimode theory (Appendix A 3).

\section{Photonic crystal cavity sample details}

The $L 3$ and $L 4 / 3$ photonic crystal cavities are fabricated by Applied Nanotools foundry via electron-beam patterning and dry etching of 220-nm-thick undoped silicon-oninsulator wafers with a $2-\mu$ m-thick buried oxide layer. To suspend the devices, the buried oxide is subsequently released via a $60 \mathrm{~s}$ timed wet etch in $49 \%$ hydrofluoric
(HF) acid. The designs are adapted from Refs. [69,70]. As shown in Fig. 2 of the main text, superimposed gratings are added to improve vertical coupling efficiency. The gratings are formed via periodic hole radii perturbations in the range $\Delta r=0 \rightarrow 0.05 r$ at a period equal to twice the lattice constant $a$. Although devices with quality factors as large as 400000 are measured for small values of $\Delta r$, the results presented in the main text use $\Delta r=0.05 r$, which significantly improves collection efficiency into our fiber-coupled detector.

\section{Experimental setup}

A more detailed version of the experimental setup depicted in the main text is provided in Fig. 9. The setup consists of a typical polarized light microscope, where the signal reflected from a $\mathrm{PhC}$ cavity is measured with balanced homodyne detection. The homodyne detector is balanced by zeroing the dc component of the homodyne signal with a digital PID feedback controller connected to a piezoactuated mirror. In this configuration, the homodyne voltage signal

$$
v_{h} \sim\left|\tilde{a}_{\text {LO }}\right|\left|\tilde{a}_{\text {cavity }}\right| \delta \phi_{\text {cavity }}(t)
$$

for a local oscillator signal $\tilde{a}_{\mathrm{LO}}$ is directly proportional to the cavity amplitude $\left|\tilde{a}_{\text {cavity }}\right|$ and to phase fluctuations 
$\delta \phi_{\text {cavity }}(t)$ resulting from the stochastic resonant frequency. An electronic spectrum analyzer is used to measure the power spectral density $S_{v v}$ of this homodyne voltage signal.

\section{Phase noise calibration}

The resonant frequency noise spectral density $S_{\omega \omega}$ can then be determined from $S_{v v}$ using the absolute calibration technique discussed in Refs. $[68,125]$. For example, consider a Mach-Zehnder interferometer with input power $P_{\text {in }}$ and splitting ratio $\eta_{h}$, which creates the in-phase local oscillator and cavity input signals

$$
\tilde{a}_{\mathrm{LO}}=\sqrt{\eta_{H} P_{\mathrm{in}}}, \quad \tilde{a}_{\mathrm{in}}=\sqrt{\left(1-\eta_{H}\right) P_{\text {in }}} .
$$

Assuming resonant drive $\left(\Delta=\omega_{L}-\omega_{0}=0\right)$ for a cavity with input power coupling rate $\Gamma_{c}$, total loss rate $\Gamma_{l}$, and a perturbative resonant frequency noise $\delta \omega(t)$, the output cavity signal is then

$$
\tilde{a}_{\mathrm{out}} \approx \sqrt{\left(1-\eta_{H}\right) P_{\mathrm{in}}} \frac{2 \Gamma_{c}-\Gamma_{l}}{\Gamma_{l}+i \delta \omega(t)},
$$

yielding a homodyne detection voltage

$$
\begin{aligned}
v_{h}(t) & \approx 2 G_{c}\left|\tilde{a}_{\mathrm{LO}}\right|\left|\tilde{a}_{\text {out }}\right| \delta \phi_{\text {cavity }}(t) \\
& \approx-2 G_{c} \sqrt{\eta_{H}\left(1-\eta_{H}\right)} P_{\mathrm{in}} \frac{2 \Gamma_{c}-\Gamma_{l}}{\Gamma_{l}^{2}} \delta \omega(t)
\end{aligned}
$$

for a detector conversion gain $G_{c}$. The final frequency noise spectral density

$$
S_{v v}^{\delta \omega} \approx \underbrace{4 G_{c}^{2} \eta_{H}\left(1-\eta_{H}\right)\left(\frac{2 \Gamma_{c}-\Gamma_{l}}{\Gamma_{l}^{2}}\right)^{2} P_{\mathrm{in}}^{2}}_{K_{\text {expt }}} S_{\omega \omega}=K_{\mathrm{expt}} S_{\omega \omega}
$$

is therefore a function of various experimental constants and cavity coupling parameters.

However, the value of $K_{\text {expt }}$ can be exactly determined by injecting a known phase noise $\delta \phi(t)=\phi_{m}\left(V_{p}\right) \cos \left(\omega_{m} t\right)$ into the interferometer with an electro-optic modulator driven with an electrical tone with frequency $\omega_{m}$ and peak voltage $V_{p}$. Under the same experimental conditions, the local oscillator and cavity input signals are, respectively,

$$
\begin{gathered}
\tilde{a}_{\mathrm{LO}}=\sqrt{\eta_{H} P_{\mathrm{in}}} e^{i \phi_{m}\left(V_{p}\right) \cos \left(\omega_{m} t\right)}, \\
\tilde{a}_{\mathrm{in}}=\sqrt{\left(1-\eta_{H}\right) P_{\mathrm{in}}} e^{i \phi_{m}\left(V_{p}\right) \cos \left(\omega_{m} t\right)} .
\end{gathered}
$$

With a small enough modulation depth $\phi_{m}\left(V_{p}\right)=\pi V_{p} / V_{\pi}$ (and, therefore, a small enough drive voltage $V_{p}$ relative to the half-wave voltage $V_{\pi}$ ), the local oscillator can be approximated to first order as

$$
\tilde{a}_{\mathrm{LO}} \approx \sqrt{\eta_{H} P_{\mathrm{in}}}\left[1+i \phi_{m}\left(V_{p}\right) \cos \left(\omega_{m} t\right)\right] .
$$

Similarly, assuming $\omega_{m} \ll \Gamma_{l}$ (as is the case in our experiment), the cavity response yields the output signal

$$
\begin{aligned}
\tilde{a}_{\text {out }} \approx & \sqrt{\left(1-\eta_{H}\right) P_{\text {in }}} \frac{2 \Gamma_{c}-\Gamma_{l}}{\Gamma_{l}} \\
& \times\left\{1+i \phi_{m}\left(V_{p}\right)\left[\cos \left(\omega_{m} t\right)+\frac{\omega_{m}}{\Gamma_{l}} \sin \left(\omega_{m} t\right)\right]\right\} .
\end{aligned}
$$

The homodyne signal

$v_{h}(t) \approx 2 G_{c} \sqrt{\eta_{H}\left(1-\eta_{H}\right)} P_{\mathrm{in}} \frac{2 \Gamma_{c}-\Gamma_{l}}{\Gamma_{l}^{2}} \omega_{m} \phi_{m}(V) \sin \left(\omega_{m} t\right)$

corresponds to a power spectral density

$$
\left.S_{v v}^{\delta \phi_{m}} \approx \underbrace{4 G_{c}^{2} \eta_{H}\left(1-\eta_{H}\right)\left(\frac{2 \Gamma_{c}-\Gamma_{l}}{\Gamma_{l}^{2}}\right)^{2} P_{\text {in }}^{2}}_{K_{\text {expt }}} \omega_{m}^{2} S_{\phi \phi}\right|_{\omega=\omega_{m}},
$$

which, similar to $S_{v v}^{\delta \omega}$, is directly proportional to $K_{\text {expt }} . K_{\text {expt }}$ can therefore be eliminated to yield an absolute calibration for the resonant frequency noise spectral density:

$$
S_{\omega \omega} \approx \frac{S_{v v}^{\delta \omega}}{K_{\mathrm{expt}}} \approx \frac{\left.\omega_{m}^{2} S_{\phi \phi}\right|_{\omega=\omega_{m}}}{\left.S_{v v}^{\delta \phi_{m}}\right|_{\omega=\omega_{m}}} S_{v v}^{\delta \omega}
$$

This result can be simplified by evaluating the phase spectral density

$$
\begin{aligned}
S_{\phi \phi}(\omega) & =\int_{-\infty}^{\infty} \phi_{m}^{2}\left(V_{p}\right)\left\langle\cos \left(\omega_{m} t\right) \cos \left[\omega_{m}(t+\tau)\right]\right\rangle e^{-i \omega \tau} d \tau \\
& =\frac{\phi_{m}^{2}\left(V_{p}\right)}{2}\left[\frac{1}{2} \delta\left(\omega-\omega_{m}\right)+\frac{1}{2} \delta\left(\omega+\omega_{m}\right)\right] \cdot \quad(\mathrm{B} 13
\end{aligned}
$$

The spectrum analyzer convolves the $\delta$ functions with the intermediate frequency filter function $F(\omega)$, which is normalized such that $F(0)=1 / \mathrm{ENBW}$ [68], where the effective noise bandwidth ENBW $=\eta_{F}$ RBW for a resolution bandwidth RBW and a filter-shape-dependent $\eta_{F} \approx 1$. Therefore, the measured noise spectral density evaluated at the modulation frequency $\omega_{m}$ becomes

$S_{\phi \phi}\left(\omega=\omega_{m}\right)=\frac{\phi_{m}^{2}\left(V_{p}\right)}{4} F(\omega) * \delta\left(\omega-\omega_{m}\right)=\frac{\phi_{m}^{2}\left(V_{p}\right)}{4 \cdot \mathrm{ENBW}}$.

Using this result, the calibration term in Eq. (B12) can be simplified to a final form 

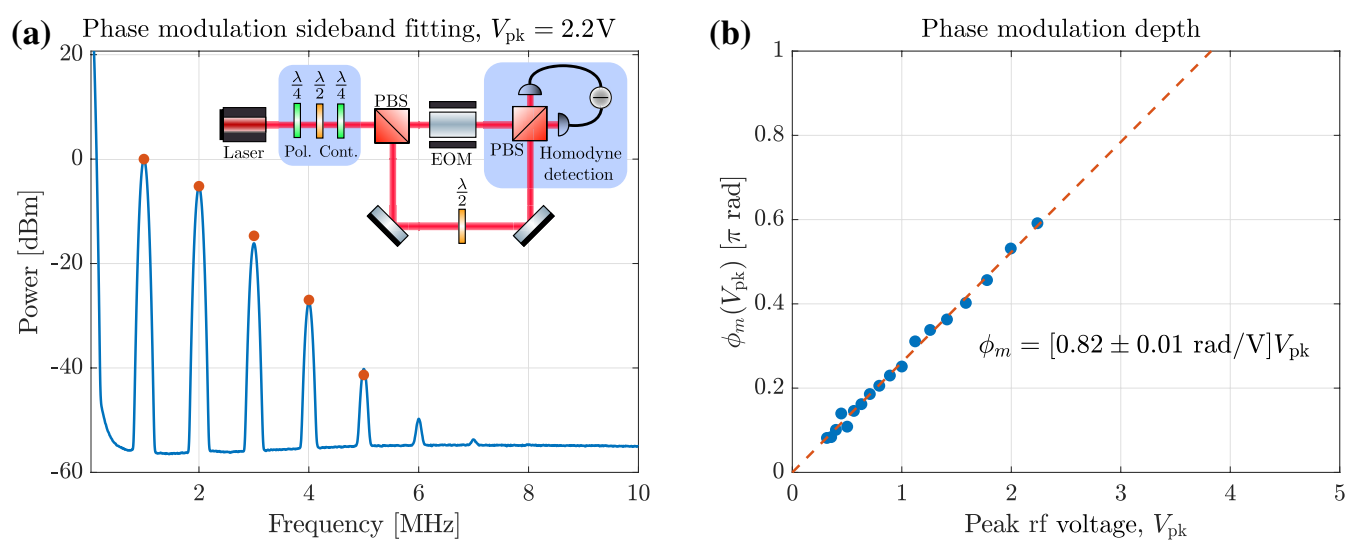

FIG. 10. Measurement of phase modulator modulation depth $\phi_{m}\left(V_{p}\right)$ at $\lambda=1550 \mathrm{~nm}$. A balanced homodyne measurement is performed on the output of a Mach-Zehnder interferometer with the EOM in one arm, yielding spectra similar to that of (a). The sideband amplitudes are fitted to find the modulation depth at each peak drive voltage $V_{p}$, and a linear fit is applied to find the modulation efficiency (b). The measured value $\phi_{m} / V_{p}=0.82 \pm 0.01 \mathrm{rad} / \mathrm{V}$ corresponds to a half-wave voltage $V_{\pi}=3.83 \mathrm{~V}$.

$$
S_{\omega \omega}(\omega)=\frac{\omega_{m}^{2} \phi_{m}^{2}\left(V_{p}\right)}{4 \eta_{F} \cdot \operatorname{RBW}} \frac{S_{v v}^{\delta \omega}(\omega)}{S_{v v}^{\delta \phi_{m}}\left(\omega_{m}\right)}
$$

that agrees with Eq. (20) of Ref. [68].

\section{a. Electo-optic phase modulator calibration}

Equation (B15) demonstrates that the calibrated frequency noise can be readily obtained by comparing the recorded rf power spectral density $S_{v v}^{\delta \omega}(\omega)$ to the calibration PSD $S_{v v}^{\delta \phi_{m}}\left(\omega_{m}\right)$ (which corresponds to a known phase spectral density) for a given calibration frequency $\omega_{m} / 2 \pi$ and spectrum analyzer RBW. The ENBW correction factor $\eta_{F}$ is a function of various spectrum analyzer settings (see Ref. [126], for example) and is therefore measured by comparing the noise marker amplitude $(\mathrm{dBm} /$ $\sqrt{\mathrm{Hz}})$ to the measured PSD divided by the RBW. This technique yields $\eta_{F} \approx 1.057$, which is approximately equal to the value given in Ref. [126] assuming typical spectrum analyzer settings.

The only remaining unknown parameter required for calibration is the peak-voltage-dependent modulation depth $\phi_{m}\left(V_{p}\right)$ of the phase modulator, which can be determined with a sideband fitting technique as shown in Fig. 10. An EOM is embedded in one arm of an unbalanced MachZehnder interferometer, yielding a homodyne signal

$$
v_{h} \propto \sum_{n} J_{n}\left(\phi_{m}\right) \cos \left(n \omega_{m} t\right)
$$

for a modulation frequency $\omega_{m}$. The power spectrum observed on the spectrum analyzer therefore consists of a periodic sequence of spectrum analyzer filter functions $F\left(\omega-n \omega_{m}\right)$ at frequencies $\omega_{n}=n \omega_{m}$ with powers proportional to $J_{n}^{2}\left(\phi_{m}\right)$. Fitting the sideband powers (relative to the $n=1$ sideband, as the $n=0$ peak is inaccessible on the ac-coupled spectrum analyzer) via a least-squares regression yields $\phi_{m}\left(V_{p}\right)$ for any peak drive voltage $V_{p}$. Figure 10(a) illustrates the result for $\lambda=1550 \mathrm{~nm}$ and $V_{p}=2.24 \mathrm{~V}$, where the Bessel functions evaluated at $\phi_{m} \approx 0.6 \pi \mathrm{rad}$ (red points) are well fitted to the measured (blue curve) peak amplitudes. After repeating the experiment for multiple values of $V_{p}$, a linear fit [Fig. 10(b)] gives the modulation efficiency

$$
\eta_{\text {mod }}=\left.\frac{\phi_{m}}{V_{p}}\right|_{\lambda_{0}=\lambda_{\text {cal }}}=0.82 \pm 0.01 \mathrm{rad} / \mathrm{V}
$$

corresponding to a half-wave voltage $V_{\pi}=3.75 \mathrm{~V}$ (roughly in line with the manufacturer-quoted value of $3.17 \mathrm{~V}$ ) at the calibration wavelength.

Note that the dc phase of the fiber interferometer in this experiment is allowed to drift, while the measurement is averaged on a timescale much longer than that of the drifta standard technique [127] which affects only the total power of the homodyne signal, not the relative magnitude of the sidebands.

\section{b. Balanced homodyne detector characterization}

Using the measured EOM modulation efficiency, the calibrated thermorefractive noise measurements in Fig. 2 of the main text are obtained by measuring the cavity reflection with the stabilized homodyne detector in Fig. 9. We confirm that the balanced photodetection is shot noise limited (with $10 \mathrm{~dB}$ of shot noise clearance) for frequencies greater than approximately $100 \mathrm{kHz}$ and balance the interferometer arms to well within $1 \mathrm{~mm}-$ over an order of magnitude shorter than the expected cavity delay (approximately centimeters). This balance is achieved by tuning a retroreflector-based delay line while observing 
TABLE I. Parameters used for calibrating the noise spectrum and computing or fitting $\Gamma_{T}$ and $V_{T}$. Independent values $n$ for $L 3$ and $L 4 / 3$ microcavities are listed as $\left\{n_{L 3}, n_{L 4 / 3}\right\}$ for cavity-dependent parameters. The mode confinement factor $\gamma_{\mathrm{Si}} \sim 1$ confirms the validity of Eq. (B15), which assumes complete confinement of the mode in silicon.

\begin{tabular}{lccc}
\hline \hline Parameter & Symbol & Value & Source \\
\hline Temperature & $T$ & $295.68 \mathrm{~K}$ & Measured \\
Si refractive index & $n_{\mathrm{Si}}$ & 3.48 & {$[128]$} \\
Si thermo-optic coefficient & $\alpha_{\mathrm{TR}}^{\mathrm{Si}}$ & $1.8 \times 10^{-4} \mathrm{~K}^{-1}$ & {$[128]$} \\
Si specific heat & $c_{V}^{\mathrm{Si}}$ & $1.64 \mathrm{~J} / \mathrm{cm}^{3} \cdot \mathrm{K}$ & {$[75]$} \\
Si thermal conductivity & $\kappa_{\mathrm{Si}}$ & $70 \mathrm{~W} / \mathrm{m} \cdot \mathrm{K}$ & {$[75]$} \\
Si thermal diffusivity (thin film) & $D^{\mathrm{Si}}$ & $0.43 \mathrm{~cm}^{2} / \mathrm{s}$ & $\kappa / c_{V}$ \\
Lattice porosity & $\phi$ & $\{0.29,0.26\}$ & Calculated \\
Patterned thermal diffusivity & $D_{\mathrm{Ti}}^{\mathrm{Si}}$ & $\{0.23,0.25\} \mathrm{cm}^{2} / \mathrm{s}$ & $D^{\mathrm{Si}}(1-\phi) /(1+\phi)[74]$ \\
Resonant wavelength & $\lambda_{0}$ & $\{1559.3,1551.5\} \mathrm{nm}$ & Measured \\
Quality factor & $Q_{l}$ & $\{168000,163000\}$ & Measured \\
Phase modulator efficiency & $\eta_{\mathrm{mod}}$ & $0.821 \mathrm{rad} / \mathrm{V}$ & Measured (1550 nm) \\
ESA noise correction factor & $\eta_{F}$ & 1.057 & Measured \\
Mode confinement factor & $\gamma_{\mathrm{Si}}$ & $\{0.96,0.95\}$ & Simulated \\
Mode volume & $\tilde{V}_{\mathrm{eff}}$ & $\{0.95,0.32\}$ & Simulated \\
Thermal mode volume & $\tilde{V}_{T}$ & $\{3.92,1.51\}$ & Simulated [Eq. (A23)] \\
\hline \hline
\end{tabular}

pulse delays from a picosecond fiber laser on both interferometer paths.

\section{Summary of experimental parameters}

Table I summarizes the various experimental parameters used to generate the data and fit parameters shown in the main text. Note that, as described in the caption of Fig. 2 of the main text, the expected thermal diffusivity is based on thermal conductivity measurements in thin silicon films [75] and the hole lattice porosity $\phi$ [74]. The porosity $\phi=3 \pi r^{2} /\left(3 \sqrt{3} a^{2} / 2-3 \pi r^{2}\right)$-calculated as the ratio of hole area $3 \pi r^{2}$ (assuming a hole radius $r$ ) to material area within a hexagonal unit cell of a lattice with lattice constant $a$-reduces the thin film diffusivity to $D_{T} \approx D(1-\phi) /$ $(1+\phi)$ [74]. This "restricted" diffusivity is used to calculate the expected decay rates in Fig. 2 of the main text.

\section{Comparison of other noise sources}

Other stochastic processes can also produce resonant frequency noise. Here, we consider two such sources: (i) multiphoton absorption leading to photothermal shot noise from free carrier recombination and (ii) self-phase modulation. Both noise sources evaluated at their respective nonlinear thresholds-as an estimate of the "worstcase" maximum noise levels - are found to be more than one order of magnitude weaker than TRN. Since the cavity is measured well within the linear regime, we find that TRN dominates both other contributions combined, thus further confirming our experimental measurements.

\section{a. Multiphoton absorption}

Multiphoton absorption (MPA) within the resonator leads to a free carrier population that stochastically recombines, producing random local heating analogous to fundamental thermorefractive noise. Considering this similarity, we can analyze the MPA photothermal shot noise by redefining the statistics of the mode-averaged temperature driving force $\bar{F}_{T}(t)$ in Eq. (A9). The mean rate of intracavity $k$-photon absorption is [81]

$$
\left\langle r_{k \mathrm{PA}}\right\rangle=\frac{\beta_{k}}{k \hbar \omega_{0}} I_{\mathrm{pk}}^{k} V_{k \mathrm{PA}},
$$

where $I_{\mathrm{pk}}=c|\tilde{a}|^{2} / 2 n V_{\text {eff }}$ is the peak intensity of the stored energy $|\tilde{a}|^{2}, \beta_{k}$ is the $k$-photon absorption coefficient, $c$ is the speed of light, and $V_{k \mathrm{PA}}=$ $\int_{\text {dielectric }}|\vec{E}(\vec{r})|^{2 k} d^{3} r / \max \left\{|\vec{E}(\vec{r})|^{2 k}\right\}$. Note that we assume that the heating produced by the photoexcited free carriers is local (i.e., no carrier diffusion). The variance of $\bar{F}_{T}(t)$ is then determined from the temperature change expected from the variance of MPA events within an infinitesimally small time (a Poisson process), yielding the autocorrelation

$$
\left\langle\bar{F}_{T, k}^{*}(t) \bar{F}_{T, k}\left(t^{\prime}\right)\right\rangle=\frac{k \hbar \omega_{0}}{c_{V}^{2} V_{T}^{2}} \beta_{k} I_{\mathrm{pk}}^{k} V_{k \mathrm{PA}} \delta\left(t-t^{\prime}\right) .
$$

Following the method of Appendix A 1, we arrive at the spectral density

$S_{\omega \omega}^{k \mathrm{PA}}(\omega)=\left(\frac{\omega_{0}}{n} \alpha_{\mathrm{TR}}\right)^{2} \frac{k \hbar \omega_{0}}{c_{V}^{2} V_{T}^{2}} \beta_{k} I_{\mathrm{pk}}^{k} V_{k \mathrm{PA}} \frac{1}{\Gamma_{T}^{2}+\omega^{2}}$,

which can be evaluated for any intracavity stored energy. Here, we consider $I_{\mathrm{pk}}$ at the nonlinear threshold, i.e., the peak intensity for a linewidth resonance shift $|\langle\Delta \omega\rangle| / 2 \Gamma_{l}=$ $\alpha_{\mathrm{TR}}\left\langle\Delta T_{k \mathrm{PA}}\right\rangle Q_{l} / n=1$. The threshold intensity can 
Comparison of frequency noise source in $\mathrm{PhC}$ cavities
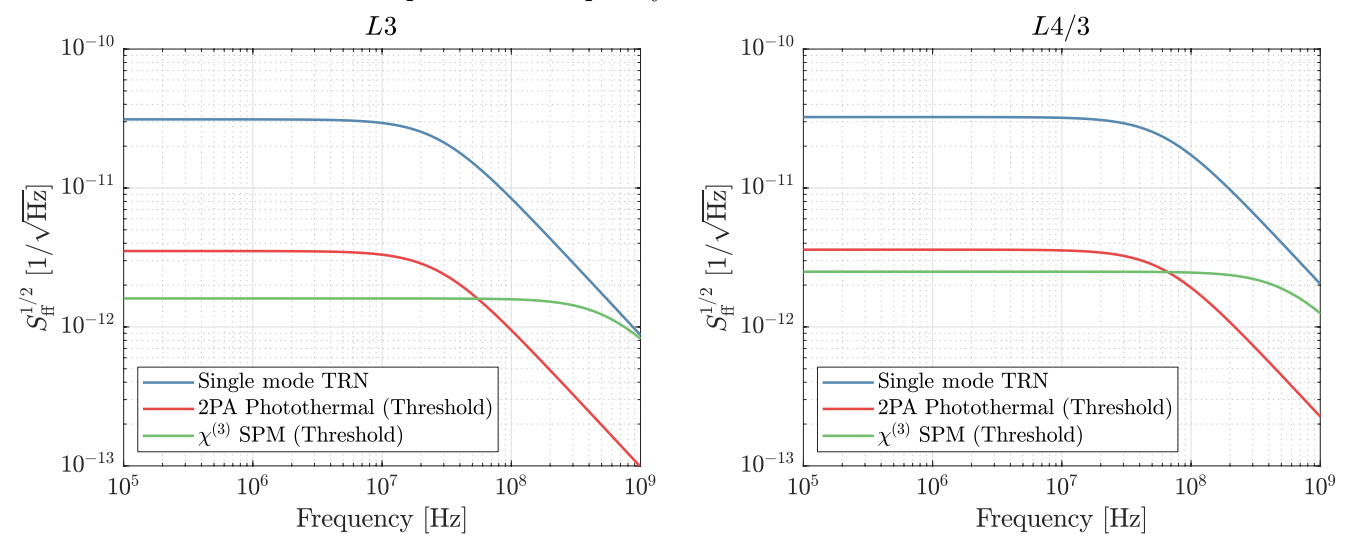

FIG. 11. Approximate spectrum of microcavity noise sources for the experimental parameters in Table I. Note that $S_{\mathrm{ff}}^{1 / 2}$ is plotted as a fractional stability (units $1 / \sqrt{\mathrm{Hz}}$ to aid comparison with cavity stabilization literature). Noises from two-photon absorption (2PA) and self-phase modulation (SPM) at their respective nonlinear threshold powers—which approximates the maximum noise level-are still smaller than TRN.

therefore be derived from the steady-state value of Eqs. (A9), which lends the average temperature change

$$
\left\langle\Delta T_{k \mathrm{PA}}\right\rangle=\frac{k \hbar \omega_{0}\left\langle r_{\mathrm{kPA}}\right\rangle}{c_{V} V_{T} \Gamma_{T}}=\frac{\beta_{k} I_{\mathrm{pk}}^{k} V_{\mathrm{kPA}}}{c_{V} \Gamma_{T} V_{T}} .
$$

Substituting this result into the spectral density equation assuming two-photon absorption as the dominant process (true for our silicon cavities driven at approximately $1550 \mathrm{~nm}$ ), we can simplify to the final result

$$
S_{\omega \omega}^{2 \mathrm{PA}, \text { threshold }}(\omega)=\frac{\omega_{0}^{2} \alpha_{\mathrm{TR}}}{n} \frac{\hbar \omega_{0}}{Q_{l} c_{V} V_{T}} \frac{2 \Gamma_{T}}{\Gamma_{T}^{2}+\omega^{2}} .
$$

This result is plotted in Fig. 11 assuming the experimental parameters of our devices listed in Table I. Comparing with Eq. (A31), we find $S_{\omega \omega}^{2 \mathrm{PA} \text {,threshold }} / S_{\omega \omega}^{\mathrm{TRN}}=$ $\left(n / Q_{l} \alpha_{\mathrm{TR}} T\right)\left(\hbar \omega_{0} / k_{B} T\right)$, which accounts for the factor of approximately 10 weaker maximum photothermal shot noise in our devices as shown in Fig. 11. We operate with an input power much lower than the nonlinear threshold power (such that $\left\langle\Delta \omega_{2 \mathrm{PA}}\right\rangle \ll \Gamma_{l}$ ), so the experimental photothermal shot noise is substantially weaker than the maximum value calculated here.

\section{b. Kerr self-phase modulation}

When confined in a $\chi^{(3)}$ nonlinear material, Poissonian fluctuations of the mean intracavity photon number impart self-phase modulational (SPM) noise on the resonant frequency. From first-order perturbation theory, the Kerr index change $\delta n(\vec{r})=3 \chi^{(3)} \epsilon(\vec{r})|\vec{E}(\vec{r})|^{2} / 8 \epsilon_{0} n^{3}$ results in a resonant frequency shift

$$
\left(\frac{\delta \omega(t)}{\omega_{0}}\right)_{\text {Kerr }}=-\frac{3 \chi^{(3)}}{4 \epsilon_{0} n^{4} V_{\text {Kerr }}} \delta|\tilde{a}(t)|^{2},
$$

where $\epsilon_{0}$ is the free space permittivity, $|\tilde{a}(t)|^{2}$ is the stored energy, and the Kerr mode volume $V_{\text {Kerr }}$ is equal to the thermal mode volume $V_{T}$ [80]. When driven with a classical source, the intracavity energy autocorrelation

$$
\left\langle\delta|\tilde{a}(t)|^{2} \delta\left|\tilde{a}\left(t^{\prime}\right)\right|^{2}\right\rangle=\frac{2 \Gamma_{c}}{\Gamma_{l}^{2}} e^{-\Gamma_{l}\left|t-t^{\prime}\right|} \hbar \omega_{0}\left\langle\left|\tilde{s}_{\text {in }}\right|^{2}\right\rangle
$$

can derived from temporal coupled mode theory assuming a constant pump power $\left\langle\left|\tilde{s}_{\text {in }}\right|^{2}\right\rangle$ coupled at rate $\Gamma_{c}$ to a cavity with composite amplitude decay rate $\Gamma_{l}$. The corresponding resonant frequency autocorrelation can then be used to compute the noise spectral density

$S_{\omega \omega}^{\mathrm{SPM}}(\omega)=\left(\frac{3 \chi^{(3)}}{4 \epsilon_{0} n^{4} V_{T}}\right)^{2}\left(\frac{4 \Gamma_{c} / \Gamma_{l}}{\Gamma_{l}^{2}+\omega^{2}}\right) \hbar \omega_{0}^{3}\left\langle\left|\tilde{s}_{\mathrm{in}}\right|^{2}\right\rangle$.

Similar to the multiphoton absorption case, we evaluate this result at the nonlinear threshold $\left\langle\Delta \omega_{\text {Kerr }}\right\rangle / 2 \Gamma_{l}=1$ for a conservative estimate of the associated noise. The final result is

$S_{\omega \omega}^{\mathrm{SPM}, \text { threshold }}(\omega)=\left(\frac{3 \chi^{(3)}}{4 \epsilon_{0} n^{4} V_{T} Q_{l}}\right)\left(\frac{2 \Gamma_{l}}{\Gamma_{l}^{2}+\omega^{2}}\right) \hbar \omega_{0}^{3}$.

Even at the threshold power, Fig. 11 shows that the SPM noise is over an order of magnitude weaker than TRN.

\section{APPENDIX C: COMPARISON OF TRN IN VARIOUS MATERIALS}

Surprisingly, the $Q_{\text {eff }}^{\max } / V_{\text {eff }}$ limits shown in Fig. 4 of the main text for several common materials lie within an order of magnitude. As shown in Table II, this observed invariance can be attributed to an inverse relationship between the thermo-optic coefficient and thermal diffusivity in 
TABLE II. Material properties used to calculate the thermal noise limits in Fig. 4 of the main text. Aluminum nitride is the only material listed with a favorable thermo-optic coefficient and thermal diffusivity.

\begin{tabular}{lccccc}
\hline \hline Material & Index $n$ & TR coefficient $\alpha_{\mathrm{TR}}\left[\mathrm{K}^{-1}\right]$ & Density $\rho\left[\mathrm{g} / \mathrm{cm}^{3}\right]$ & Heat capacity $c_{V}[\mathrm{~J} / \mathrm{g} \cdot \mathrm{K}]$ & $\mathrm{Thermal} \mathrm{diffusivity} D_{T}\left[\mathrm{~cm}{ }^{2} / \mathrm{s}\right]$ \\
\hline $\mathrm{Si}$ & 3.48 & $1.8 \times 10^{-4}$ & 2.32 & 0.7 & 0.8 \\
$\mathrm{GaAs}$ & 3.38 & $2.35 \times 10^{-4}$ & 5.32 & 0.35 & 0.31 \\
$\mathrm{InP}$ & 3.16 & $2 \times 10^{-4}$ & 4.81 & 0.31 & 0.37 \\
$\mathrm{Si}_{3} N_{4}$ & 1.99 & $2.5 \times 10^{-5}$ & 4.65 & 0.7 & 0.02 \\
$\mathrm{LiNbO}_{3}$ & 2.21 & $3.2 \times 10^{-5}$ & 5.32 & 0.63 & $7 \times 10^{-3}$ \\
$\mathrm{AlN}$ & 2.19 & $3 \times 10^{-5}$ & 3.23 & 0.6 & 1.47 \\
\hline \hline
\end{tabular}

common materials. Yet this relationship is not fundamental: aluminum nitride, for example, is shown to outperform all other plotted materials by over an order of magnitude due to its simultaneously large thermal conductivity and small thermo-optic coefficient. This realization demonstrates the importance of material choice when designing state-of-theart high- $Q / V_{\text {eff }}$ resonators.

\section{APPENDIX D: EFFECTS OF TRN ON ALL-OPTICAL QUBITS}

\section{Derivation of qubit coupling strengths}

This section derives the figures of merit for qubit operation in nonlinear optical cavities. For more information, see Refs. [57,95]. The procedure is to first derive the classical equations of motion for fields in nonlinear oscillators and then to quantize them, deriving the Hamiltonian and the single-photon coupling strength. In classical cavity electrodynamics, a cavity field can be expressed as a sum of resonant modes

$$
E(\vec{x}, t)=\sum_{\omega} C_{\omega}\left[A_{\omega}(t) E_{\omega}(\vec{x}) e^{-i \omega t}+\text { c.c. }\right],
$$

where $C_{\omega}=\sqrt{\hbar \omega / 2 \epsilon_{0}}$. The modes $E_{\omega}$ satisfy the Helmholtz equation $\nabla \times\left(\nabla \times E_{\omega}\right)=\left(n^{2} \omega^{2} / c^{2}\right) E_{\omega}$. This form is a generalized eigenvalue equation, and the resulting solutions can be orthogonalized: $\int n^{2} E_{\omega^{\prime}}^{*} E_{\omega} d^{3} \vec{r}=c^{2} \int B_{\omega^{\prime}}^{*} B_{\omega} d^{3} \vec{r}=$ $\delta_{\omega^{\prime} \omega}$. With this normalization, we find that the electromagnetic energy density in the cavity is $U=\sum_{\omega} \hbar \omega\left|A_{\omega}\right|^{2}$. Therefore, $A_{\omega}$ is the normalized field operator, where $\left|A_{\omega}\right|^{2}$ gives the number of photons in the mode $E_{\omega}$.

Nonlinear interactions can be treated as perturbations, because the nonlinearity is weak on the order of a single optical cycle. The Helmholtz equation acquires a nonlinear polarization $P=\epsilon_{0}\left(\chi^{(2)}: E^{2}+\chi^{(3)}: E^{3}+\cdots\right)$, which can be integrated to give perturbations to the equations of motion for $A_{\omega}$ [129]:

$$
\begin{array}{r}
\nabla \times(\nabla \times E)+\frac{n^{2}}{c^{2}} \frac{\partial^{2} E}{\partial t^{2}}=-\frac{1}{c^{2}} \frac{\partial^{2}\left(P / \epsilon_{0}\right)}{\partial t^{2}} \\
\Rightarrow \frac{d A_{\omega}}{d t}=\frac{i \omega}{2 C_{\omega}} \int E_{\omega}(\vec{x})^{*}\left[\frac{P(\vec{x}, t)}{\epsilon_{0}}\right]_{\omega} e^{i \omega t} d^{3} \vec{r}
\end{array}
$$

\section{a. $\operatorname{Kerr}\left(\chi^{(3)}\right)$ interaction}

In the $\chi^{(3)}$ case, we have a cavity with a single resonant mode $E_{\omega}$. The polarization term due to the Kerr interaction is $P=\epsilon_{0} \chi^{(3)}:\left(C_{\omega} A_{\omega} E_{\omega} e^{-i \omega t}+\text { c.c. }\right)^{3}$. This term gives rise to the equation of motion $\dot{A}_{\omega}=-i \chi\left|A_{\omega}\right|^{2} A_{\omega}$, where

$\chi=-\frac{3 \hbar \omega^{2} \chi^{(3)}}{4 n^{4} \epsilon_{0}} \frac{1}{V_{\mathrm{Kerr}}}, \quad V_{\mathrm{Kerr}} \equiv \frac{\left(\int n^{2}\left|E_{\omega}\right|^{2} d^{3} \vec{r}\right)^{2}}{\int_{*} n^{4}\left|E_{\omega}\right|^{4} d^{3} \vec{r}}$

Quantizing the field to satisfy the commutation relations $\left[\hat{A}_{\omega}, \hat{A}_{\omega}^{\dagger}\right]=1$, this equation of motion can be generated from the Hamiltonian:

$$
H_{\mathrm{Kerr}}=\frac{1}{2} \chi \hat{A}_{\omega}^{\dagger} \hat{A}_{\omega}^{\dagger} \hat{A}_{\omega} \hat{A}_{\omega}
$$

As an open quantum system, the field interacts with a bath through Lindblad dissipation terms, in this case $L=\sqrt{2 \Gamma} A_{\omega}$, where $\Gamma=\omega / 2 Q$. The figure of merit for strong coupling is

$$
\mathrm{FOM}_{\chi^{(3)}}=\frac{\chi}{2 \Gamma}=\underbrace{\frac{3 \pi \hbar c}{2 n \epsilon_{0}} \frac{\chi^{(3)}}{\lambda^{4}}}_{K_{\chi}} \frac{Q}{\tilde{V}_{\mathrm{Kerr}}} .
$$

\section{b. Second-order $\left(\chi^{(2)}\right)$ interaction}

In this case, we have two fields at frequencies $(\omega, 2 \omega)$. The polarization term is $P=\epsilon_{0} \chi^{(2)}:\left(C_{\omega} A_{\omega} E_{\omega} e^{-i \omega t}+\right.$ $C_{2 \omega} A_{2 \omega} E_{2 \omega} e^{-2 i \omega t}+$ c.c. $)^{2}$, which gives rise to the equations

$$
\dot{A}_{2 \omega}=-\frac{1}{2} \epsilon A_{\omega}^{2}, \quad \dot{A}_{\omega}=\epsilon A_{2 \omega} A_{\omega}^{*},
$$

where

$$
\begin{gathered}
\epsilon=\frac{\omega \sqrt{\hbar \omega / \epsilon_{0}}}{n^{3} V_{\text {shg }}^{1 / 2}} \chi^{(2)}, \\
V_{\text {shg }}=\frac{\left(\int n^{2}\left|E_{2 \omega}\right|^{2} d^{3} \vec{r}\right)\left(\int n^{2}\left|E_{\omega}\right|^{2} d^{3} \vec{r}\right)^{2}}{\left|\int_{*} n^{3} E_{2 \omega}^{*} E_{\omega} E_{\omega} d^{3} \vec{r}\right|^{2}}
\end{gathered}
$$


TABLE III. Silicon material properties assumed to calculate the qubit figures of merit at $\lambda_{0}=2.3 \mu \mathrm{m}$ and $T=300 \mathrm{~K}$.

\begin{tabular}{lccc}
\hline \hline Parameter & Symbol & Value & Source \\
\hline$\chi^{(3)}$ nonlinear index & $n_{2}$ & $1.2 \times 10^{-13} \mathrm{~cm}^{2} / \mathrm{W}$ & [130], Sec. 11 \\
$\chi^{(3)}$ FOM constant & $K_{\chi}$ & $8.7 \times 10^{-11}$ & Calculated [Eq. (D6)] \\
$\chi^{(2)}(\mathrm{dc} E$-field induced $)$ & $\chi^{(2)}$ & $40 \mathrm{pm} / \mathrm{V}$ & [108] \\
$\chi^{(2)}$ FOM constant & $K_{\epsilon}$ & $1.3 \times 10^{-7}$ & Calculated [Eq. (D13)] \\
Thermo-optic coefficient & $\alpha_{\mathrm{TR}}^{\mathrm{Si}}$ & $1.8 \times 10^{-4} \mathrm{~K}^{-1}$ & {$[128]$} \\
Thermal diffusivity & $D^{\mathrm{Si}}$ & $0.8 \mathrm{~cm}^{2} / \mathrm{s}$ & [75] \\
\hline \hline
\end{tabular}

in the case that $\vec{E}$ and $\vec{P}$ are aligned (otherwise, $\epsilon$ is reduced by a geometric factor). The integral $\int(\ldots) d^{3} \vec{r}$ is taken over all space, while $\int_{*}(\ldots) d^{3} \vec{r}$ is restricted to the nonlinear material.

As before, we can quantize the fields $\hat{A}_{\omega}$ and $\hat{A}_{2 \omega}$ and derive a Hamiltonian corresponding to Eqs. (D7). As an open quantum system, there will also be Lindblad dissipation terms $\Gamma_{1}=\omega / 2 Q_{1}$ and $\Gamma_{2}=2 \omega / 2 Q_{2}$ :

$$
\begin{gathered}
H=\epsilon \frac{\hat{A}_{2 \omega}^{\dagger} \hat{A}_{\omega} \hat{A}_{\omega}-\hat{A}_{\omega}^{\dagger} \hat{A}_{\omega}^{\dagger} \hat{A}_{2 \omega}}{2 i}, \\
L_{1}=\sqrt{2 \Gamma_{1}} A_{\omega}, \\
L_{2}=\sqrt{2 \Gamma_{2}} A_{2 \omega} .
\end{gathered}
$$

The figure of merit for strong coupling again is expressed as a ratio of the coupling rate $\epsilon$ to the loss rate. Here, there are two loss channels, and a common approach is to take the geometric mean of the two (a choice motivated by the limit $Q_{2} \ll Q_{1}$, in which the $\chi^{(2)}$ interaction can be adiabatically eliminated to a $\chi^{(3)}$ one with $\left.\chi / \Gamma \propto \epsilon^{2} / \Gamma_{1} \Gamma_{2}\right)$. Thus, we set the figure of merit to be

$\operatorname{FOM}_{\chi^{(2)}}=\frac{\epsilon}{2 \bar{\Gamma}}=\frac{\epsilon}{2 \sqrt{\Gamma_{1} \Gamma_{2}}}=\underbrace{\sqrt{\frac{\pi \hbar c}{n^{3} \epsilon_{0}}} \frac{\chi^{(2)}}{\lambda^{2}}}_{K_{\epsilon}} \frac{\sqrt{Q_{1} Q_{2}}}{\tilde{V}_{\text {shg }}^{1 / 2}}$.

In the main text, we assume $Q_{1}=Q_{2}=Q$ such that $\mathrm{FOM}_{\chi^{(2)}} \propto Q / V_{\text {shg }}^{1 / 2}$.

\section{Parameters}

The parameters used to generate Fig. 5 of the main text are included in Table III.

[1] LIGO Scientific Collaboration, GW170817: Observation of Gravitational Waves from a Binary Neutron Star Inspiral, Phys. Rev. Lett. 119, 161101 (2017).
[2] S. P. Yu, J. A. Muniz, C. L. Hung, and H. J. Kimble, TwoDimensional Photonic Crystals for Engineering AtomLight Interactions, Proc. Natl. Acad. Sci. U.S.A. 116, 12743 (2019).

[3] U. Delić, M. Reisenbauer, K. Dare, D. Grass, V. Vuletić, N. Kiesel, and M. Aspelmeyer, Cooling of a Levitated Nanoparticle to the Motional Quantum Ground State, Science 367, 892 (2020).

[4] H. B. Callen and T. A. Welton, Irreversibility and Generalized Noise, Phys. Rev. 83, 34 (1951).

[5] Y. Levin, Internal Thermal Noise in the LIGO Test Masses: A Direct Approach, Phys. Rev. D 57, 659 (1998).

[6] LIGO Scientific Collaboration, Sensitivity of the Advanced LIGO Detectors at the Beginning of Gravitational Wave Astronomy, Phys. Rev. D 93, 112004 (2016).

[7] G. D. Cole, W. Zhang, M. J. Martin, J. Ye, and M. Aspelmeyer, Tenfold Reduction of Brownian Noise in HighReflectivity Optical Coatings, Nat. Photonics 7, 644 (2013).

[8] D. Heinert, S. Kroker, D. Friedrich, S. Hild, E. B. Kley, S. Leavey, I. W. Martin, R. Nawrodt, A. Tünnermann, S. P. Vyatchanin, and K. Yamamoto, Calculation of Thermal Noise in Grating Reflectors, Phys. Rev. D 88, 042001 (2013).

[9] S. Kroker, J. Dickmann, C. B. R. Hurtado, D. Heinert, R. Nawrodt, Y. Levin, and S.P. Vyatchanin, Brownian Thermal Noise in Functional Optical Surfaces, Phys. Rev. D 96, 022002 (2017).

[10] K. Numata, A. Kemery, and J. Camp, Thermal-Noise Limit in the Frequency Stabilization of Lasers with Rigid Cavities, Phys. Rev. Lett. 93, 250602 (2004).

[11] T. Kessler, T. Legero, and U. Sterr, Thermal Noise in Optical Cavities Revisited, J. Opt. Soc. Am. B 29, 178 (2012).

[12] N. O. Zhadnov, K. S. Kudeyarov, D. S. Kryuchkov, I. A. Semerikov, K. Y. Khabarova, and N. N. Kolachevsky, On the Thermal Noise Limit of Ultrastable Optical Cavities, Quantum Electron. 48, 425 (2018).

[13] K. J. Vahala, Optical Microcavities, Nature (London) 424, 839 (2003).

[14] A. M. Armani, R. P. Kulkarni, S. E. Fraser, R. C. Flagan, and K. J. Vahala, Label-Free, Single-Molecule Detection with Optical Microcavities, Science 317, 783 (2007).

[15] T. J. Kippenberg, R. Holzwarth, and S. A. Diddams, Microresonator-Based Optical Frequency Combs, Science 332, 555 (2011).

[16] T. J. Kippenberg, A. L. Gaeta, M. Lipson, and M. L. Gorodetsky, Dissipative Kerr Solitons in Optical Microresonators, Science 361, eaan8083 (2018). 
[17] D. T. Spencer, T. Drake, T. C. Briles, J. Stone, L. C. Sinclair, C. Fredrick, Q. Li, D. Westly, B. R. Ilic, A. Bluestone et al., An Optical-Frequency Synthesizer Using Integrated Photonics, Nature (London) 557, 81 (2018).

[18] A. Dutt, C. Joshi, X. Ji, J. Cardenas, Y. Okawachi, K. Luke, A. L. Gaeta, and M. Lipson, On-Chip Dual-Comb Source for Spectroscopy, Sci. Adv. 4, e1701858 (2018).

[19] E. Obrzud, M. Rainer, A. Harutyunyan, M. H. Anderson, J. Liu, M. Geiselmann, B. Chazelas, S. Kundermann, S. Lecomte, M. Cecconi et al., A Microphotonic Astrocomb, Nat. Photonics 13, 31 (2019).

[20] M. Eichenfield, R. Camacho, J. Chan, K. J. Vahala, and O. Painter, A Picogram- and Nanometre-Scale PhotonicCrystal Optomechanical Cavity, Nature (London) 459, 550 (2009).

[21] A. Goban, C. L. Hung, S. P. Yu, J. D. Hood, J. A. Muniz, J. H. Lee, M. J. Martin, A. C. McClung, K. S. Choi, D. E. Chang, O. Painter, and H. J. Kimble, Atom-Light Interactions in Photonic Crystals, Nat. Commun. 5, 3808 (2014).

[22] J. M. Kindem, A. Ruskuc, J. G. Bartholomew, J. Rochman, Y. Q. Huan, and A. Faraon, Control and Single-Shot Readout of an Ion Embedded in a Nanophotonic Cavity, Nature (London) 580, 201 (2020).

[23] M. Li, H. Liang, R. Luo, Y. He, J. Ling, and Q. Lin, Photon-Level Tuning of Photonic Nanocavities, Optica 6 , 860 (2019).

[24] H. Cao and J. Wiersig, Dielectric Microcavities: Model Systems for Wave Chaos and Non-Hermitian Physics, Rev. Mod. Phys. 87, 61 (2015).

[25] Z. Gao, S. T. M. Fryslie, B. J. Thompson, P. S. Carney, and K. D. Choquette, Parity-Time Symmetry in Coherently Coupled Vertical Cavity Laser Arrays, Optica 4, 323 (2017).

[26] W. Chen, Ş. K. Özdemir, G. Zhao, J. Wiersig, and L. Yang, Exceptional Points Enhance Sensing in an Optical Microcavity, Nature (London) 548, 192 (2017).

[27] S. Mittal, E. A. Goldschmidt, and M. Hafezi, A Topological Source of Quantum Light, Nature (London) 561, 502 (2018).

[28] J. K. Jang, A. Klenner, X. Ji, Y. Okawachi, M. Lipson, and A. L. Gaeta, Synchronization of Coupled Optical Microresonators, Nat. Photonics 12, 688 (2018).

[29] F. Monifi, J. Zhang, Ş. K. Özdemir, B. Peng, Y.-x. Liu, F. Bo, F. Nori, and L. Yang, Optomechanically Induced Stochastic Resonance and Chaos Transfer between Optical Fields, Nat. Photonics 10, 399 (2016).

[30] M. Evans, S. Ballmer, M. Fejer, P. Fritschel, G. Harry, and G. Ogin, Thermo-Optic Noise in Coated Mirrors for HighPrecision Optical Measurements, Phys. Rev. D 78, 102003 (2008).

[31] L. D. Landau and E. M. Lifshitz, Statistical Physics (Elsevier, New York, 1980).

[32] C. Kittel, Temperature Fluctuation: An Oxymoron, Phys. Today 41, No. 5, 93 (1988).

[33] B. B. Mandelbrot, Temperature Fluctuation: A WellDefined and Unavoidable Notion, Phys. Today 42, No. 1, 71 (1989).

[34] M. Falcioni, D. Villamaina, A. Vulpiani, A. Puglisi, and A. Sarracino, Estimate of Temperature and Its Uncertainty in Small Systems, Am. J. Phys. 79, 777 (2011).
[35] T. C. P. Chui, D. R. Swanson, M. J. Adriaans, J. A. Nissen, and J. A. Lipa, Temperature Fluctuations in the Canonical Ensemble, Phys. Rev. Lett. 69, 3005 (1992).

[36] L. Stodolsky, Temperature Fluctuations in Multiparticle Production, Phys. Rev. Lett. 75, 1044 (1995).

[37] J. Hickman and Y. Mishin, Temperature Fluctuations in Canonical Systems: Insights from Molecular Dynamics Simulations, Phys. Rev. B 94, 184311 (2016).

[38] T. Jahnke, S. Lanéry, and G. Mahler, Operational Approach to Fluctuations of Thermodynamic Variables in Finite Quantum Systems, Phys. Rev. E 83, 011109 (2011).

[39] P. Seifert, X. Lu, P. Stepanov, J. R. Durán Retamal, J. N. Moore, K.-C. Fong, A. Principi, and D. K. Efetov, MagicAngle Bilayer Graphene Nanocalorimeters: Toward Broadband, Energy-Resolving Single Photon Detection, Nano Lett. 20, 3459 (2020).

[40] B. Karimi, F. Brange, P. Samuelsson, and J. P. Pekola, Reaching the Ultimate Energy Resolution of a Quantum Detector, Nat. Commun. 11, 367 (2020).

[41] K. Wanser, Fundamental Phase Noise Limit in Optical Fibres due to Temperature Fluctuations, Electron. Lett. 28, 53 (1992).

[42] R. Moeller and W. Burns, Observation of Thermal Noise in a Dynamically Biased Fiber-Optic Gyro, Opt. Lett. 21, 171 (1996).

[43] S. Foster, A. Tikhomirov, and M. Milnes, Fundamental Thermal Noise in Distributed Feedback Fiber Lasers, IEEE J. Quantum Electron. 43, 378 (2007).

[44] L. Duan, General Treatment of the Thermal Noises in Optical Fibers, Phys. Rev. A 86, 023817 (2012).

[45] M. L. Gorodetsky and I. S. Grudinin, Fundamental Thermal Fluctuations in Microspheres, J. Opt. Soc. Am. B 21, 697 (2004).

[46] A. B. Matsko, A. A. Savchenkov, N. Yu, and L. Maleki, Whispering-Gallery-Mode Resonators as Frequency References. I. Fundamental Limitations, J. Opt. Soc. Am. B 24, 1324 (2007).

[47] J. Lim, A. A. Savchenkov, E. Dale, W. Liang, D. Eliyahu, V. Ilchenko, A. B. Matsko, L. Maleki, and C. W. Wong, Chasing the Thermodynamical Noise Limit in WhisperingGallery-Mode Resonators for Ultrastable Laser Frequency Stabilization, Nat. Commun. 8, 8 (2017).

[48] G. Huang, E. Lucas, J. Liu, A. S. Raja, G. Lihachev, M. L. Gorodetsky, N. J. Engelsen, and T. J. Kippenberg, Thermorefractive Noise in Silicon-Nitride Microresonators, Phys. Rev. A 99, 061801(R) (2019).

[49] K. Saurav and N. Le Thomas, Probing the Fundamental Detection Limit of Photonic Crystal Cavities, Optica 4, 757 (2017).

[50] S. Jiang, S. H. Pan, S. S. Deka, C. Y. Fang, Z. Chen, Y. Fainman, and A. El Amili, Impact of Fundamental Temperature Fluctuations on the Frequency Stability of Metallo-dielectric Nanolasers, IEEE J. Quantum Electron. 55, 2000910 (2019).

[51] W. Jin, Q.-F. Yang, L. Chang, B. Shen, H. Wang, M. A. Leal, L. Wu, A. Feshali, M. Paniccia, K. J. Vahala, and J. E. Bowers, Hertz-Linewidth Semiconductor Lasers Using CMOS-Ready Ultra-high-Q Microresonators, arXiv: 2009.07390 . 
[52] T. E. Drake, J. R. Stone, T. C. Briles, and S. B. Papp, Thermal Decoherence and Laser Cooling of Kerr Microresonator Solitons, Nat. Photonics 14, 480 (2020).

[53] T. Asano, Y. Ochi, Y. Takahashi, K. Kishimoto, and S. Noda, Photonic Crystal Nanocavity with a $Q$ Factor Exceeding Eleven Million, Opt. Express 25, 1769 (2017).

[54] S. Hu, M. Khater, R. Salas-Montiel, E. Kratschmer, S. Engelmann, W. M. Green, and S. M. Weiss, Experimental Realization of Deep-Subwavelength Confinement in Dielectric Optical Resonators, Sci. Adv. 4, eaat2355 (2018).

[55] M. Geitner, F. Aguilar Sandoval, E. Bertin, and L. Bellon, Low Thermal Fluctuations in a System Heated out of Equilibrium, Phys. Rev. E 95, 032138 (2017).

[56] K. Komori, Y. Enomoto, H. Takeda, Y. Michimura, K. Somiya, M. Ando, and S. W. Ballmer, Direct Approach for the Fluctuation-Dissipation Theorem under Nonequilibrium Steady-State Conditions, Phys. Rev. D 97, 102001 (2018).

[57] H. Mabuchi, Qubit Limit of Cavity Nonlinear Optics, Phys. Rev. A 85, 015806 (2012).

[58] J. D. Joannopoulos, S. G. Johnson, J. N. Winn, and R. D. Meade, Photonic Crystals: Molding the Flow of Light (Princeton University, Princeton, NJ, 2008).

[59] Y. Zhang, J. Moser, J. Güttinger, A. Bachtold, and M. I. Dykman, Interplay of Driving and Frequency Noise in the Spectra of Vibrational Systems, Phys. Rev. Lett. 113, 255502 (2014).

[60] R. P. Feynman and A. R. Hibbs, Quantum Mechanics and Path Integrals (McGraw-Hill, New York, 1965).

[61] X. Sun, R. Luo, X. C. Zhang, and Q. Lin, Squeezing the Fundamental Temperature Fluctuations of a High-Q Microresonator, Phys. Rev. A 95, 023822 (2017).

[62] M. Notomi, Manipulating Light with Strongly Modulated Photonic Crystals, Rep. Prog. Phys. 73, 096501 (2010).

[63] Y. Zhang and M. Lončar, Submicrometer Diameter Micropillar Cavities with High Quality Factor and Ultrasmall Mode Volume, Opt. Lett. 34, 902 (2009).

[64] T. J. Kippenberg, S. M. Spillane, and K. J. Vahala, Demonstration of Ultra-high-Q Small Mode Volume Toroid Microcavities on a Chip, Appl. Phys. Lett. 85, 6113 (2004).

[65] H. Lee, T. Chen, J. Li, K. Y. Yang, S. Jeon, O. Painter, and K. J. Vahala, Chemically Etched Ultrahigh-Q WedgeResonator on a Silicon Chip, Nat. Photonics 6, 369 (2012).

[66] F. Ding, T. Stöferle, L. Mai, A. Knoll, and R. F. Mahrt, Vertical Microcavities with High-q and Strong Lateral Mode Confinement, Phys. Rev. B 87, 161116(R) (2013).

[67] M. Galli, S. L. Portalupi, M. Belotti, L. C. Andreani, L. O'Faolain, and T. F. Krauss, Light Scattering and Fano Resonances in High-Q Photonic Crystal Nanocavities, Appl. Phys. Lett. 94, 071101 (2009).

[68] M. L. Gorodetsky, A. Schliesser, G. Anetsberger, S. Deleglise, and T.J. Kippenberg, Determination of the Vacuum Optomechanical Coupling Rate Using Frequency Noise Calibration, Opt. Express 18, 23236 (2010).

[69] M. Minkov and V. Savona, Automated Optimization of Photonic Crystal Slab Cavities, Sci. Rep. 4, 5124 (2015).

[70] M. Minkov, V. Savona, and D. Gerace, Photonic Crystal Slab Cavity Simultaneously Optimized for Ultra-high $Q / V$ and Vertical Radiation Coupling, Appl. Phys. Lett. 111, 131104 (2017).

[71] Nguyen-Vi-Quynh Tran, S. Combrié, and A. De Rossi, Directive Emission from High-Q Photonic Crystal Cavities through Band Folding, Phys. Rev. B 79, 041101(R) (2009).

[72] E. Gavartin, R. Braive, I. Sagnes, O. Arcizet, A. Beveratos, T. J. Kippenberg, and I. Robert-Philip, Optomechanical Coupling in a Two-Dimensional Photonic Crystal Defect Cavity, Phys. Rev. Lett. 106, 203902 (2011).

[73] Y. Levin, Fluctuation-Dissipation Theorem for Thermorefractive Noise, Phys. Lett. A 372, 1941 (2008).

[74] A. Jain, Y. J. Yu, and A. J. H. McGaughey, Phonon Transport in Periodic Silicon Nanoporous Films with Feature Sizes Greater than 100 nm, Phys. Rev. B 87, 195301 (2013).

[75] J. Cuffe, J. K. Eliason, A. A. Maznev, K. C. Collins, J. A. Johnson, A. Shchepetov, M. Prunnila, J. Ahopelto, C. M. Sotomayor Torres, G. Chen, and K. A. Nelson, Reconstructing Phonon Mean-Free-Path Contributions to Thermal Conductivity Using Nanoscale Membranes, Phys. Rev. B 91, 245423 (2015).

[76] W. Weng, J. D. Anstie, T. M. Stace, G. Campbell, F. N. Baynes, and A. N. Luiten, Nano-Kelvin Thermometry and Temperature Control: Beyond the Thermal Noise Limit, Phys. Rev. Lett. 112, 160801 (2014).

[77] W. Weng, P. S. Light, and A. N. Luiten, Ultra-sensitive Lithium Niobate Thermometer Based on a Dual-Resonant Whispering-Gallery-Mode Cavity, Opt. Lett. 43, 1415 (2018).

[78] T. Lu, H. Lee, T. Chen, S. Herchak, J.-H. Kim, S. E. Fraser, R. C. Flagan, and K. Vahala, High Sensitivity Nanoparticle Detection Using Optical Microcavities, Proc. Natl. Acad. Sci. U.S.A. 108, 5976 (2011).

[79] W. Loh, A. A. Green, F. N. Baynes, D. C. Cole, F. J. Quinlan, H. Lee, K. J. Vahala, S. B. Papp, and S. A. Diddams, Dual-Microcavity Narrow-Linewidth Brillouin Laser, Optica 2, 225 (2015).

[80] H. Choi, M. Heuck, and D. Englund, Self-Similar Nanocavity Design with Ultrasmall Mode Volume for SinglePhoton Nonlinearities, Phys. Rev. Lett. 118, 223605 (2017).

[81] C. Panuski, M. Pant, M. Heuck, R. Hamerly, and D. Englund, Single Photon Detection by Cavity-Assisted AllOptical Gain, Phys. Rev. B 99, 205303 (2019).

[82] W. H. Pernice, C. Xiong, C. Schuck, and H. X. Tang, High$Q$ Aluminum Nitride Photonic Crystal Nanobeam Cavities, Appl. Phys. Lett. 100, 091105 (2012).

[83] J. Zhu, S. K. Ozdemir, Y. F. Xiao, L. Li, L. He, D. R. Chen, and L. Yang, On-Chip Single Nanoparticle Detection and Sizing by Mode Splitting in an Ultrahigh-Q Microresonator, Nat. Photonics 4, 46 (2010).

[84] S. M. Spillane, T. J. Kippenberg, K. J. Vahala, K. W. Goh, E. Wilcut, and H. J. Kimble, Ultrahigh-Q Toroidal Microresonators for Cavity Quantum Electrodynamics, Phys. Rev. A 71, 013817 (2005).

[85] T. Asano and S. Noda, Optimization of Photonic Crystal Nanocavities Based on Deep Learning, Opt. Express 26, 32704 (2018). 
[86] F. Alpeggiani, L. C. Andreani, and D. Gerace, Effective Bichromatic Potential for Ultra-high Q-Factor Photonic Crystal Slab Cavities, Appl. Phys. Lett. 107, 261110 (2015).

[87] Q. Quan and M. Loncar, Deterministic Design of Wavelength Scale, Ultra-high Q Photonic Crystal Nanobeam Cavities, Opt. Express 19, 18529 (2011).

[88] S. Hu and S. M. Weiss, Design of Photonic Crystal Cavities for Extreme Light Concentration, ACS Photonics 3, 1647 (2016).

[89] Y. Lai, S. Pirotta, G. Urbinati, D. Gerace, M. Minkov, V. Savona, A. Badolato, and M. Galli, Genetically Designed L3 Photonic Crystal Nanocavities with Measured Quality Factor Exceeding One Million, Appl. Phys. Lett. 104, 241101 (2014).

[90] K. Y. Yang, D. Y. Oh, S. H. Lee, Q. F. Yang, X. Yi, B. Shen, H. Wang, and K. Vahala, Bridging Ultrahigh-Q Devices and Photonic Circuits, Nat. Photonics 12, 297 (2018).

[91] F. Wang, R. E. Christiansen, Y. Yu, J. Mørk, and O. Sigmund, Maximizing the Quality Factor to Mode Volume Ratio for Ultra-small Photonic Crystal Cavities, Appl. Phys. Lett. 113, 241101 (2018).

[92] G. Moille, X. Lu, A. Rao, Q. Li, D. A. Westly, L. Ranzani, S. B. Papp, M. Soltani, and K. Srinivasan, Kerr-Microresonator Soliton Frequency Combs at Cryogenic Temperatures, Phys. Rev. Applied 12, 034057 (2019).

[93] M. Heuck, K. Jacobs, and D. R. Englund, ControlledPhase Gate Using Dynamically Coupled Cavities and Optical Nonlinearities, Phys. Rev. Lett. 124, 160501 (2020).

[94] M. Heuck, K. Jacobs, and D. R. Englund, Photon-Photon Interactions in Dynamically Coupled Cavities, Phys. Rev. A 101, 042322 (2020).

[95] S. Krastanov, M. Heuck, J. H. Shapiro, P. Narang, D. R. Englund, and K. Jacobs, Room-Temperature Photonic Logical Qubits via Second-Order Nonlinearities, arXiv:2002.07193 [Nat. Commun. (to be published)].

[96] M. Li, Y.-L. Zhang, H. X. Tang, C.-H. Dong, G.-C. Guo, and C.-L. Zou, Photon-Photon Quantum Phase Gate in a Photonic Molecule with $\chi^{(2)}$ Nonlinearity, Phys. Rev. Applied 13, 044013 (2020).

[97] R. Yanagimoto, T. Onodera, E. Ng, L. G. Wright, P. L. McMahon, and H. Mabuchi, Engineering a Kerr-Based Deterministic Cubic Phase Gate via Gaussian Operations, Phys. Rev. Lett. 124, 240503 (2020).

[98] Q. Zhao, L. Zhang, and O. D. Miller, Minimum DielectricResonator Mode Volumes, arXiv:2008.13241.

[99] J. Lu, M. Li, C.-L. Zou, A. A. Sayem, and H. X. Tang, Towards $1 \%$ Single-Photon Anharmonicity with Periodically Poled Lithium Niobate Microring Resonators, Optica 7, 1654 (2020).

[100] T. Tiecke, J. D. Thompson, N. P. de Leon, L. Liu, V. Vuletić, and M. D. Lukin, Nanophotonic Quantum Phase Switch with a Single Atom, Nature (London) 508, 241 (2014).

[101] D. Najer, I. Söllner, P. Sekatski, V. Dolique, M. C. Löbl, D. Riedel, R. Schott, S. Starosielec, S. R. Valentin, A. D. Wieck et al., A Gated Quantum Dot Strongly Coupled to an Optical Microcavity, Nature (London) 575, 622 (2019).
[102] R. E. Evans, M. K. Bhaskar, D. D. Sukachev, C. T. Nguyen, A. Sipahigil, M. J. Burek, B. Machielse, G. H. Zhang, A. S. Zibrov, E. Bielejec et al., Photon-Mediated Interactions between Quantum Emitters in a Diamond Nanocavity, Science 362, 662 (2018).

[103] S. Wehner, D. Elkouss, and R. Hanson, Quantum Internet: A Vision for the Road Ahead, Science 362, eaam9288 (2018).

[104] A. V. Kuhlmann, J. Houel, A. Ludwig, L. Greuter, D. Reuter, A. D. Wieck, M. Poggio, and R. J. Warburton, Charge Noise and Spin Noise in a Semiconductor Quantum Device, Nat. Phys. 9, 570 (2013).

[105] S. Ferretti and D. Gerace, Single-Photon Nonlinear Optics with Kerr-Type Nanostructured Materials, Phys. Rev. B 85, 033303 (2012).

[106] W. T. M. Irvine, K. Hennessy, and D. Bouwmeester, Strong Coupling between Single Photons in Semiconductor Microcavities, Phys. Rev. Lett. 96, 057405 (2006).

[107] A. Majumdar and D. Gerace, Single-Photon Blockade in Doubly Resonant Nanocavities with Second-Order Nonlinearity, Phys. Rev. B 87, 235319 (2013).

[108] E. Timurdogan, C. V. Poulton, M. J. Byrd, and M. R. Watts, Electric Field-Induced Second-Order Nonlinear Optical Effects in Silicon Waveguides, Nat. Photonics 11, 200 (2017).

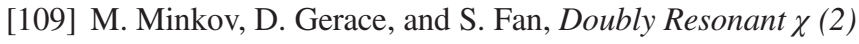
Nonlinear Photonic Crystal Cavity Based on a Bound State in the Continuum, Optica 6, 1039 (2019).

[110] W. Loh, S. Yegnanarayanan, F. O'Donnell, and P. W. Juodawlkis, Ultra-narrow Linewidth Brillouin Laser with Nanokelvin Temperature Self-Referencing, Optica 6, 152 (2019).

[111] H. J. Kimble, B. L. Lev, and J. Ye, Optical Interferometers with Reduced Sensitivity to Thermal Noise, Phys. Rev. Lett. 101, 260602 (2008).

[112] M. L. Gorodetsky, Thermal Noises and Noise Compensation in High-Reflection Multilayer Coating, Phys. Lett. A 372, 6813 (2008).

[113] T. Chalermsongsak, E. D. Hall, G. D. Cole, D. Follman, F. Seifert, K. Arai, E. K. Gustafson, J. R. Smith, M. Aspelmeyer, and R. X. Adhikari, Coherent Cancellation of Photothermal Noise in GaAs/ $\mathrm{Al}_{0.92} \mathrm{Ga}_{0.08}$ As Bragg Mirrors, Metrologia 53, 860 (2016).

[114] Z. Zhang, P. Zhao, P. Lin, and F. Sun, Thermo-Optic Coefficients of Polymers for Optical Waveguide Applications, Polymer 47, 4893 (2006).

[115] X. Gan, H. Clevenson, and D. Englund, Polymer Photonic Crystal Nanocavity for Precision Strain Sensing, ACS Photonics 4, 1591 (2017).

[116] S. G. Johnson, M. Ibanescu, M. A. Skorobogatiy, O. Weisberg, J. D. Joannopoulos, and Y. Fink, Perturbation Theory for Maxwell's Equations with Shifting Material Boundaries, Phys. Rev. E 65, 066611 (2002).

[117] J. S. Jensen and O. Sigmund, Topology Optimization for Nano-photonics, Laser Photonics Rev. 5, 308 (2011).

[118] P. Cheben, R. Halir, J. H. Schmid, H. A. Atwater, and D. R. Smith, Subwavelength Integrated Photonics, Nature (London) 560, 565 (2018).

[119] J. Park, S. K. Ozdemir, F. Monifi, T. Chadha, S. H. Huang, P. Biswas, and L. Yang, Titanium Dioxide Whispering Gallery Microcavities, Adv. Opt. Mater. 2, 711 (2014). 
[120] T. Handa, H. Tahara, T. Aharen, and Y. Kanemitsu, Large Negative Thermo-Optic Coefficients of a Lead Halide Perovskite, Sci. Adv. 5, eaax0786 (2019).

[121] V. B. Braginsky, M. L. Gorodetsky, and S. P. Vyatchanin, Thermodynamical Fluctuations and Photo-thermal Shot Noise in Gravitational Wave Antennae, Phys. Lett. A 264, 1 (1999).

[122] V. B. Braginsky, M. L. Gorodetsky, and S. P. Vyatchanin, Thermo-refractive Noise in Gravitational Wave Antennae, Phys. Lett. A 271, 303 (2000).

[123] W. Zhang, F. Baynes, S. A. Diddams, and S. B. Papp, Microrod Optical Frequency Reference in the Ambient Environment, Phys. Rev. Applied 12, 024010 (2019).

[124] C. W. Gardiner, Adiabatic Elimination in Stochastic Systems. I. Formulation of Methods and Application to Few-Variable Systems, Phys. Rev. A 29, 2814 (1984).
[125] A. Schliesser, R. Rivière, G. Anetsberger, O. Arcizet, and T. J. Kippenberg, Resolved-Sideband Cooling of a Micromechanical Oscillator, Nat. Phys. 4, 415 (2008).

[126] Keysight Technologies, Spectrum and Signal Analyzer Measurements and Noise, Application Note No. 5966, 2017.

[127] H. Ludvigsen, M. Tossavainen, and M. Kaivola, Laser Linewidth Measurements Using Self-Homodyne Detection with Short Delay, Opt. Commun. 155, 180 (1998).

[128] J. Komma, C. Schwarz, G. Hofmann, D. Heinert, and R. Nawrodt, Thermo-Optic Coefficient of Silicon at $1550 \mathrm{~nm}$ and Cryogenic Temperatures, Appl. Phys. Lett. 101, 041905 (2012).

[129] R. W. Boyd, Nonlinear Optics (Academic, New York, 2019).

[130] R. Hamerly, Coherent LQG Control, Free-Carrier Oscillations, Optical Ising Machines and Pulsed OPO Dynamics, arXiv:1608.07551. 